\title{
Spatial Processing of Visual Information in the Movement-Detecting Pathway of the Fly
}

\author{
Characteristics and Functional Significance
}

\author{
M.V. Srinivasan ${ }^{1,2}$ and D.R. Dvorak ${ }^{1, *}$ \\ Departments of Neurobiology ${ }^{1}$ and Applied Mathematics ${ }^{2}$, Australian National University, Canberra, ACT 2600, Australia
}

Accepted May 30, 1980

Summary. 1. Spatial processing of visual signals in the fly's movement-detecting pathway was studied by recording the responses of directionally-selective movement-detecting (DSMD) neurons in the lobula plate. The summarized results pertain to a type of neuron which preferentially responds to horizontal movement directed toward the animal's midline. Three kinds of visual stimuli were used: moving gratings, reversing-contrast gratings and reversing-contrast bars.

2. Contrast-sensitivity functions were measured for reversing-contrast gratings. With horizontally-oriented gratings, sensitivity is maximum at the lowspatial-frequency end and falls off toward high frequencies. With vertically-oriented gratings, sensitivity is maximum at an intermediate spatial frequency (Fig. 7). These results are consistent with a neural organization in which the DSMD neuron receives its input through an array of small-field ("sampling") units, each unit having a receptive field comprising an excitatory centre and horizontally-extending inhibitory flanks (Fig. 17).

3. Threshold contrast functions were measured for reversing-contrast bars (Figs. 11 and 12). The results for horizontally-oriented bars differ from those for vertically-oriented bars in a way that is consistent with the hypothesized neural organization.

4. Response to horizontally-moving, verticallyoriented gratings of various spatial frequencies were measured (Figs. 13 and 14) and the results used to infer the azimuthal angle $\widetilde{\Delta \phi}$ between the visual axes of sampling units participating in directionally-selective movement detection (Fig. 18). At a mean luminance of $10 \mathrm{~cd} / \mathrm{m}^{2}$, the inferred value of $\widetilde{\Delta \phi}$ is approximately equal to the angle between the visual axes of

\footnotetext{
* Present address: University of Michigan, Medical School, Department of Physiology, Ann Arbor, Michigan 48109, USA

Abbreviation: DSMD, directionally-selective movement detector
}

adjacent ommatidia of a horizontal row, in the frontal eye region (Figs. 14, 18).

5. When the level of ambient light is decreased, the response characteristics of the DSMD neuron change in a way which suggests that, within the eye, the neural representation of the visual scene becomes coarser than the ommatidial mosaic. When mean luminance is lowered by $3 \log$ units (from $10 \mathrm{~cd} / \mathrm{m}^{2}$ to $0.01 \mathrm{~cd} / \mathrm{m}^{2}$ ) the altered response characteristics suggest neuronal modifications such that the excitatory centres of the sampling units' receptive fields become $50 \%$ wider (Figs. 7 and 17), the inhibitory flanks become weaker and more diffuse, and $\widetilde{\Delta \phi}$ increases by $30 \%$ (Figs. 14 and 18 ). Neuronal mechanisms that might mediate such changes are proposed and discussed.

6. The experimentally-measured characteristics of the DSMD neuron are compared with theoreticallypredicted characteristics of an ideal movement detector, designed for optimum performance. This comparison suggests that the fly's movement-detecting pathway prefilters visual signals in such a way as to extract the most reliable movement cues, and that it analyzes the filtered information in a way that achieves maximum directional selectivity. The characteristics of the movement-detecting pathway vary with luminance in a way that ensures the best attainable performance at each level of ambient light (Fig. 21).

\section{Introduction}

The lobula plate of the fly contains neurons which are exquisitely sensitive to movement. These neurons are few in number, they have large visual fields typically covering the entire eye - and their responses are selective to direction of movement (Bishop et al., 
1968; Dvorak et al., 1975; Hausen, 1976). They can be broadly divided into two groups, those responding to motion in the horizontal direction, and those responding to motion in the vertical direction (Bishop et al., 1968). It is believed that the neurons that respond to horizontal movement are involved in visually-guided course control, detecting deviations from the intended course, and issuing corrective commands to the flight control system (Bishop and Keehn, 1967; Eckert, 1980).

The sensitivity of these neurons to movement, the directional selectivity of the responses, and the relative ease with which responses can be recorded, makes these neurons attractive subjects in which to study the filtering and analysis of visual information by a neural pathway that is specialized to detect specifically-directed movement.

This study describes how visual information is spatially filtered prior to detecting movement, and how the filtered information is subsequently analyzed to determine direction of motion. It also examines luminance-dependent changes in the characteristics of movement detection, and attempts to interpret these changes in terms of neuronal circuitry. The functional significance of the experimentally observed characteristics is explored by comparing them with those expected from an ideal directionally-selective movement detector, designed for optimum performance.

\section{Materials and Methods}

\section{Stimuli}

Visual stimuli were created on the screen of a Tektronix 604 CRT display oscilloscope, connected to a DEC PDP 11-03 digital computer via a home-built display interface. The interface created a 1,024 line raster on the CRT on which gratings or bars, oriented parallel to the line elements of the raster, were displayed. The intensity of each line element could be set to any one of 800 different levels. 12 adjoining line elements together create a bar with a width of one degree of visual angle.

Three kinds of stimulus were used:

(a) Reversing-contrast grating. This is a grating with a spatially sinusoidal intensity-profile, which reverses its contrast abruptly and periodically in time.

(b) Moving grating. This is a grating with a spatially sinusoidal intensity-profile, which drifts to the left or to the right at constant speed.

(c) Reversing-contrast bar. This is a bar with an intensity that varies sinusoidally with time, above and below a mean intensity equal to that of the background.

For gratings, the variable parameters are:

(i) Contrast, C, defined as $\frac{I_{\max }-I_{\min }}{I_{\max }+I_{\min }}$, where $I_{\max }$ and $I_{\min }$ are the intensities of the peak and trough respectively of the sinusoidal intensity-profile of the grating. Contrast could be varied from 0 to 1 in steps of 0.0025 . (ii) Spatial frequency $v$, in cycles per degree, defined as the angular spatial frequency at the centre of the grating, as seen by the fly, $v$ could be varied from 0 to 6.4 cycles/degree in steps of 0.0125 . Geometrical distortion of the grating, arising from the fact that the screen is not curved around the fly but flat, causes the angular spatial frequency of the grating as seen by the fly, to increase by $25 \%$ in going from the centre to the periphery of the screen.

(iii) Temporal frequency, $\mathrm{f}$, in $\mathrm{Hz}$. In the case of a reversing contrast grating, it specifies the frequency of contrast reversals; e.g. $5 \mathrm{~Hz}$ implies 10 reversals/s. In the case of a moving grating, it specifies the temporal frequency of (sinusoidal) intensity fluctuations created by the moving grating at a fixed point in space. $v$ and $\mathrm{f}$ together determine the angular speed of the grating, which is given by ( $\mathrm{f} / v)$ degrees/s.

For bars, the variable parameters are:

(i) Contrast, $C$, defined as before, with $I_{\max }$ and $I_{\min }$ now referring to the maximum and minimum intensities of the bar as the intensity varies sinusoidally in time.

(ii) Width of bar W, in degrees.

(iii) Temporal frequency, $\mathrm{f}$, in $\mathrm{Hz}$. This specifies the frequency at which the intensity of the bar fluctuates in time.

For all types of stimuli, the mean luminance of the screen, $\mathrm{L}$, defined as $\frac{I_{\max }+I_{\min }}{2}$, was set at $10 \mathrm{~cd} / \mathrm{m}^{2}$. This level ensured that operation of the phosphor was linear to within $3 \%$ for all contrasts ranging from 0 to 1 . The mean luminance was lowered, if desired, by placing neutral density filters between the CRT and the eye. The CRT had a P31 phosphor with an emission spectrum peaking at $535 \mathrm{~nm}$ and with half-intensity points at $500 \mathrm{~nm}$ and $565 \mathrm{~nm}$, measured in photometric units (Dvorak et al., 1980) This spectrum is well suited for stimulating the R1-6 photoreceptors, which are thought to provide the principal input to the movement-detecting neurons (Bishop, 1969; Kirschfeld, 1972; McCann and Arnett, 1972; Lillywhite and Dvorak, in press).

The experimental fly was positioned so that its eyes were $10 \mathrm{~cm}$ in front of the screen of the CRT. For most of the experiments, the screen was fitted with a circular aperture $10 \mathrm{~cm}$ in diameter, so that the grating subtended a visual angle of $53^{\circ}$. In special cases, specifically mentioned in the text, the aperture was reduced to $5 \mathrm{~cm}$, corresponding to a subtense of $28^{\circ}$.

The display CRT was mounted on a stand which enabled rotation about an axis perpendicular to the face of the CRT, passing through the centre of the aperture. Thus, the stimulus could be presented at any desired orientation. In addition, orthogonal orientations could be obtained by a switching arrangement which interchanged the $X$ and $Y$ input signals of the CRT.

\section{Preparation and Recording}

Experiments were performed on female specimens of the sheep blowfly, Lucilia sericata, 7 to 28 days post-emergence. The anaesthetized fly was secured to a small mound of dental periphery wax resting on a stand. The legs, wings and halteres were immobilized with beeswax. A small area of exoskeleton, covering the posterior part of the left optic lobe, was removed with a microscalpel, and the underlying fatty tissue carefully pushed aside to expose a small portion of the posterior surface of the optic lobe. A tungsten microclectrode (Hubel, 1957) was used to record extracellular action potentials. The electrode was positioned with reference to the branching pattern of the tracheae, which provided reliable landmarks and enabled us to probe the same region of the lobula plate in each preparation.

After a single unit was isolated and its preferred direction noted, a high contrast test grating was used to find the most responsive part of the unit's receptive field. The animal was then 
left undisturbed for $15-20 \mathrm{~min}$, during which time it viewed a blank field with luminance equal to the mean luminance of the visual stimuli that were to follow. Experiments were then commenced if the preparation showed no signs of becoming unstable. If the mean luminance was manipulated during the course of an experiment, a waiting period of $20 \mathrm{~min}$ was used to allow the eye to adapt to the new level of luminance. During this period the eye viewed a blank field at the new level of luminance. Further details of preparation and recording can be found in Dvorak et al. (1980)

\section{Data Analysis and Measurement of Threshold Contrast}

Action potentials from the isolated unit were amplified, displayed on an oscilloscope, and fed into the computer for analysis. Each experimental run typically consisted of 4 trials, at the end of which the computer produced either an ensemble-averaged histogram, or a cumulative histogram, of action potential occurrence versus time. The duration of a trial, the number of trials in the run, and the number of bins in the histogram could be selected by the experimenter.

In the experiments with moving gratings, each experimental trial typically consisted of four 5-s periods: (i) stationary grating, (ii) movement in the null direction, (iii) stationary grating, (iv) movement in the preferred direction. The computer counted the number of spikes that occurred during each 5 -s period. In the experiments with reversing-contrast gratings, each trial typically consisted of an initial 5-s period during which the grating did not change, followed by another 5 -s period during which it reversed at the specified temporal frequency. In the experiments with reversing-contrast bars, each trial typically consisted of an initial 5-s period during which only the background was displayed (i.e. a blank field), followed by a 5 -s period during which the intensitymodulated bar was superimposed upon the background.

In each case, the response of the neuron was measured as the average number of spikes that occurred during the 5-s stimulus period, minus the average number of spikes that occurred spontaneously during the preceding 5 -s period in which there was no stimulus. In some instances 10 -s periods were used (e.g. Fig. 5).

Threshold contrast was defined as the contrast required to evoke a criterion response, and contrast sensitivity was defined as the reciprocal of threshold contrast. A criterion response of 10 spikes was used in all the measurements of contrast sensitivity reported here. Preliminary experiments revealed no significant differences in the shapes of the measured contrast-sensitivity functions when half or double the usual criterion was used.

In measuring contrast sensitivity functions, spatial frequencies were chosen in random order, and measurements at a standard spatial frequency (usually 0.05 cycles/degree) were frequently interleaved in order to ensure that the preparation was stable and sensitivity had not changed.

\section{Results}

\section{The Experimental Neuron}

In their survey of the lobula plate Bishop et al. (1968) reported eight classes of directionally-selective motion-detecting units. Cells were grouped according to their receptive field location (ipsilateral or contralateral) and direction of preferred movement (up or down, right-to-left, or left-to-right). In the early stages of the present study these functional classes were regularly encountered. However, after sampling many animals, it became obvious that one type of neuron was consistently the easiest to isolate and produced the largest extracellular spikes $(400-700 \mu \mathrm{V})$. This unit, a type IIal according to the scheme of Bishop et al. (1968), was maximally sensitive to horizontal, lateralto-medial movement presented anywhere in the lower $60-70 \%$ of the contralateral compound eye. The cell had a specific recording locus which was recognizable with reference to the pattern of tracheal branching on the posterior surface of the lobula plate. Responses from this neuron were always associated with spikes of smaller amplitude from a unit sensitive to downward movement presented ipsilaterally.

Considering the position of our recording electrode in the lobula plate, and the results of recent double-electrode experiments by Eckert (in prep.), we believe that our recordings are from the $\mathrm{Hl}$ neuron first described by Hausen (1976). There are only two such cells in the fly's visual system. They form a symmetrical pair, one having its visual field in the left eye, the other in the right eye. Whether or not our recordings were actually from this cell exclusively can be unequivocally answered only by dye injection experiments, which were not part of the present investigation. Nevertheless, the data from different preparations are sufficiently consistent that we feel justified in assuming that our results represent a physiologically homogeneous population of neurons, if not a single neuron. All of the experiments described in this paper were performed on this type of neuron, except where specifically indicated otherwise. For convenience, we shall refer to our experimental neuron as the Hl DSMD (directionally selective movement detector), bearing in mind the above qualification.

Bishop et al. (1968) reported that the movement sensitivity of the type IIal unit has a broad peak approximately $50-70^{\circ}$ in diameter, centered on the frontal part of the compound eye slightly below the equator. This finding was confirmed by preliminary experiments in the present study. In the results that follow, each animal was positioned such that the $53^{\circ}$ diameter stimulus was approximately centered upon the peak of this sensitivity profile.

The average response histogram of Fig. 1 illustrates the response of the H1 DSMD when the stimulus is a vertically-oriented grating moving horizontally, with contrast well above threshold. The histogram shows the result of averaging 10 repetitions of a stimulus sequence consisting of the following phases: stationary, movement in preferred direction, stationary, movement in null direction. During the stationary period, the neuron fires spontaneously at a rate of 


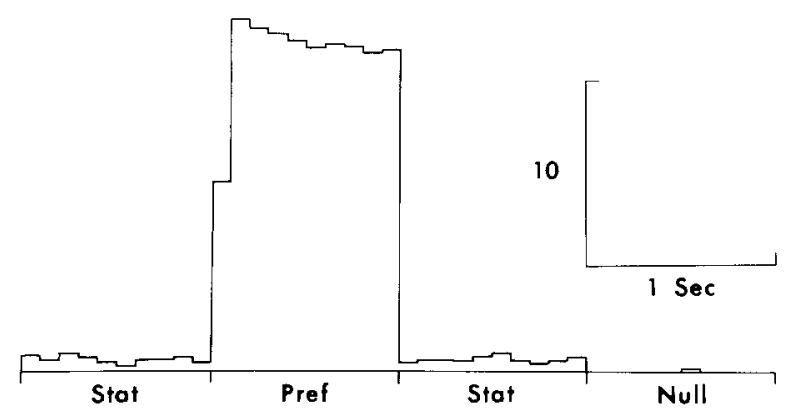

Fig. 1. Response of the HI DSMD to motion in the preferred and null directions. The stimulus is a vertically-oriented grating, which undergoes four consecutive 1-s phases: (i) stationary, (ii) movement in preferred direction, (iii) stationary, (iv) movement in null direction. Ensemble average of 10 trials. Stimulus parameters: $L=10 \mathrm{~cd} / \mathrm{m}^{2}, C=0.94, v=0.05$ cycles/degree, $f=1.17 \mathrm{~Hz}$

3-8 spikes/s. When the grating drifts in the preferred direction a maintained unmodulated discharge at frequencies approaching $200 \mathrm{~Hz}$ is elicited. When the grating drifts in the null direction the spontaneous discharge of the neuron is suppressed.

\section{Directional Selectivity}

Figure 2 illustrates directional selectivity of the $\mathrm{Hl}$ DSMD by showing a polar plot of evoked response versus direction of movement. The response depends upon direction, and is maximum when motion is inward (i.e. toward the midline) and directed approximately $10^{\circ}$ below horizontal. It would appear that the response of the HI DSMD encodes the vector component of motion along the preferred direction. The dashed circle shows, for comparison, the response that would theoretically be expected from a neuron which signals the vector component of motion along this direction (H). Bishop et al. (1968) report similar results for a type IIal unit. In all experiments pertaining to the HI DSMD, we shall refer to the preferred direction as "horizontal" and the direction perpendicular to it as "vertical", with the understanding that these directions are $10^{\circ}$ away from the true horizontal and vertical, respectively.

Directional selectivity of the HI DSMD is poor when the contrast of the grating is low. This is illustrated in Fig. 3. At very low contrast, motion in either the preferred or the null direction causes the neuron's firing rate to rise above the spontaneous level. This direction-insensitive response is not evoked by motion per se, but rather by the temporal fluctuations of intensity (flicker) that each photoreceptor sees as the grating moves across its visual field. At threshold contrast, the response of the neuron is dominated by this direction-insensitive component. As the contrast of the grating is increased, the response includes

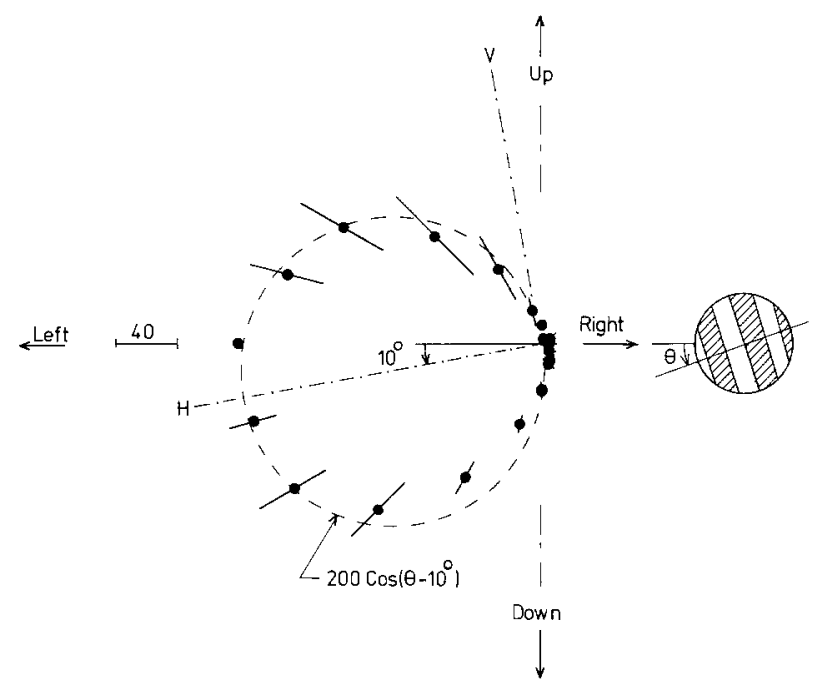

Fig. 2. Directional selectivity of the HI DSMD. The stimulus is a grating, moving along a direction perpendicular to its orientation. Parameters: $L=10 \mathrm{~cd} / \mathrm{m}^{2}, \quad C=0.1, \quad v=0.05$ cycles/degree, $f=$ $1.17 \mathrm{~Hz}$. The figure shows a polar plot of response versus direction of movement. The response is the average, over 4 trials, of the number of spikes that occur during a 5 -s period in which the grating moves. Data pooled from 4 animals (mean \pm s.d.), where the data from each animal is normalized to 200 spikes along the horizontal leftward direction. Bar: 40 spikes. Dot-dashed line, labelled $\mathrm{H}$, indicates approximate preferred direction (leftward, $10^{\circ}$ below horizontal). Dot-dashed line labelled $\mathrm{V}$ is perpendicular to $\mathrm{H}$. Dashed circle depicts the function $200 \cos \left(\Theta-10^{\circ}\right)$. It represents the response theoretically expected from a detector which signals the component of movement along the preferred direction (H)

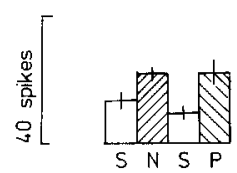

Contrast $\rightarrow \quad 0.04$

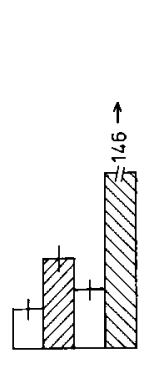

0.10

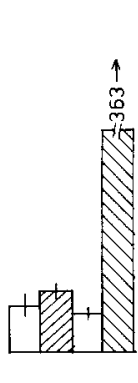

0.20

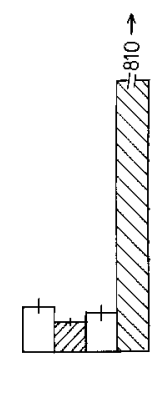

0.30
Fig. 3. Directional selectivity of the H1 DSMD at various contrasts. Stimulus is a vertically oriented grating which undergoes four consecutive 5-s phases: (i) stationary (S), (ii) movement in null direction $(N)$, (iii) stationary $(S)$, (iv) movement in preferred direction $(P)$. Bins show number of spikes occurring during each phase (mean \pm s.d., 4 trials)

a direction-sensitive component which becomes increasingly prominent and dominates the neuron's response at high contrast.

\section{Reversing-Contrast Gratings}

The direction-insensitive component of the neuron's response can be isolated by using a stimulus in which 


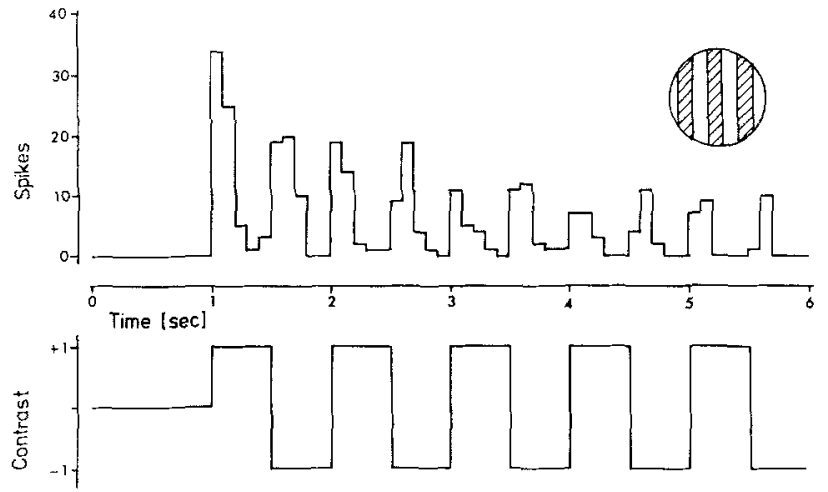

Fig. 4. Histogram of response evoked in HI DSMD by a vertically oriented reversing-contrast grating, accumulated over 8 trials. Stimulus parameters: $L=10 \mathrm{~cd} / \mathrm{m}^{2}, C=1.0, v=0.05$ cycles $/$ degree, $f=$ $1.0 \mathrm{~Hz}$. Lower trace: instants of time at which the grating reverses contrast

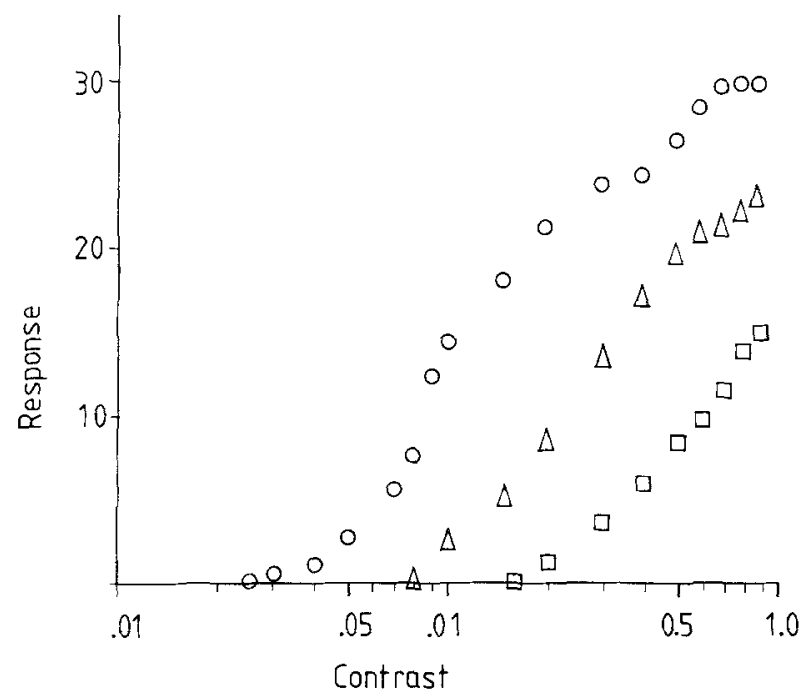

Fig. 5. Response-versus-contrast functions for a HI DSMD, measured using vertically-oriented reversing-contrast gratings of three different spatial frequencies: 0.0125 cycles/degree (squares), 0.025 cycles/degree (triangles), 0.1 cycles/degree (circles). For all gratings $L=10 \mathrm{~cd} / \mathrm{m}^{2}, f=1.0 \mathrm{~Hz}$. Response is measured as described in "Methods", using 5-s bins. Each data point represents the mean of 4 measurements

there is temporal variation of intensity, but no movement (Geiger and Poggio, 1975). One such stimulus is a grating which is stationary, but reverses its contrast periodically in time. Figure 4 shows the response evoked by a reversing-contrast grating. The response is phasic in nature. Each reversal of contrast produces a transient burst of impulses, and there is some habituation of the response during the first few reversals.

The magnitude of the response increases with the contrast of the grating. This is illustrated in Fig. 5, which shows response-versus-contrast functions for gratings of three different spatial frequencies. Re-

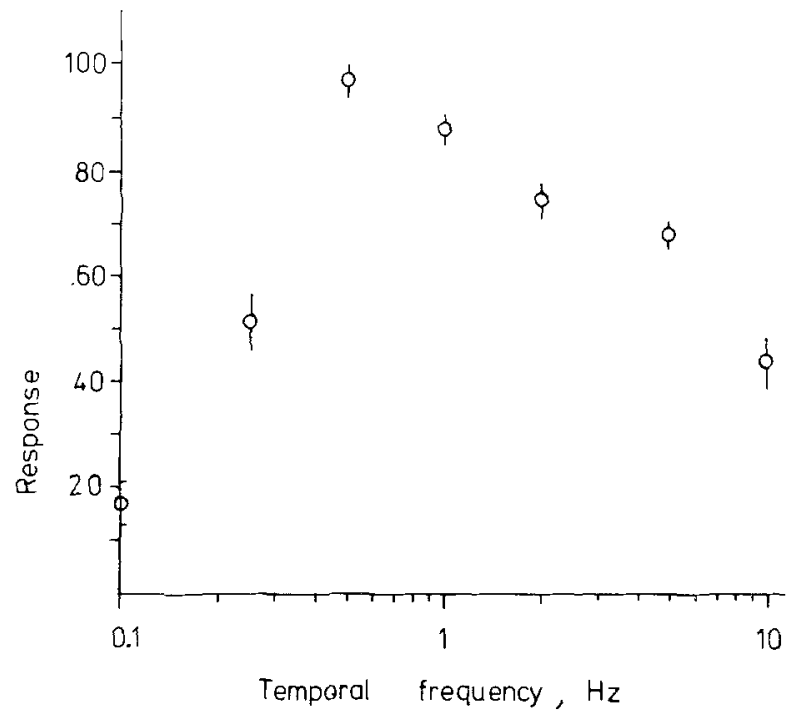

Fig. 6. Response-versus-temporal frequency for a H1 DSMD, measured using a vertically oriented reversing-contrast grating. Stimulus parameters are: $L=10 \mathrm{~cd} / \mathrm{m}^{2}, C=1.0, \nu=0.1$ cycles $/$ degree. Response measured as described in "Methods" using 10-s bins. Each data point shows mean and s.d. of 4 measurements

sponse is measured as described in "Methods". In the example of Fig. 5, the curves for the three frequencies are similar in shape, but displaced from each other along the contrast axis. We did not investigate whether responses evoked by gratings of different spatial frequencies are indistinguishable when they are equal as defined by our response criterion.

The magnitude of the response also depends upon the temporal frequency of contrast reversals, being maximum in the vicinity of $0.5 \mathrm{~Hz}$ (Fig. 6).

Figure 7 shows contrast-sensitivity functions of the Hl DSMD measured using reversing-contrast gratings that are oriented vertically (filled circles) or horizontally (open circles). (The exact orientations are as indicated by the dashed lines labelled $\mathrm{V}$ and $\mathrm{H}$ on the polar response plot of Fig. 2). Contrast sensitivity is defined as the reciprocal of threshold contrast, and the contrast-sensitivity function is a plot of contrast sensitivity versus spatial frequency. Threshold contrast is defined as the contrast that is required to achieve a criterion response of 10 spikes, and is measured as described in "Methods".

The upper set of contrast-sensitivity functions was measured at the highest level of mean luminance $\left(10 \mathrm{~cd} / \mathrm{m}^{2}\right)$. With vertically-oriented gratings, sensitivity is maximum at an intermediate spatial frequency (in the vicinity of 0.1 cycles/degree) and falls off toward low and high frequencies. With horizontallyoriented gratings there is no intermediate-frequency peak; sensitivity is maximum at the low-frequency end, and falls off at high frequencies. At the low- 


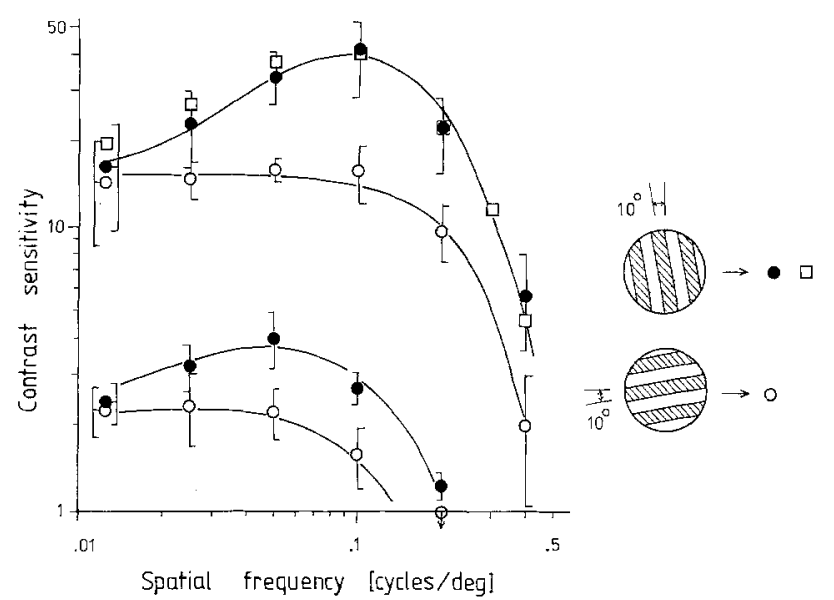

Fig. 7. Contrast sensitivity functions of HI DSMD, measured using reversing-contrast gratings (circles) oriented along the directions labelled $H$ and $V$ in Fig. 2. The two upper functions were measured at $L=10 \mathrm{~cd} / \mathrm{m}^{2}$; they represent data pooled from 10 animals. The two lower functions were measured at $L=0.01 \mathrm{~cd} / \mathrm{m}^{2}$; they represent data pooled from 5 animals. Filled circles: measurements made with vertically-oriented gratings $(V)$; open circles: measurements with horizontally-oriented gratings $(H)$ (see Fig. 2). $f=$ $1.0 \mathrm{~Hz}$ in all cases. The solid curves through the data points represent the theoretical contrast-sensitivity function of a receptive-field model with an excitatory centre and inhibitory flanks, in which the parameters have been adjusted to obtain a least-squares fit to the contrast-sensitivity data. Details in Appendix A. Squares represent measurements of contrast sensitivity made by using a vertically-oriented $(V)$ grating that moves in the preferred direction, with $L=10 \mathrm{~cd} / \mathrm{m}^{2}$ and $f=1.17 \mathrm{~Hz}$. They represent data pooled from 5 animals

frequency end, sensitivity is independent of grating orientation, as expected. In the limiting case of zero spatial frequency (d.c.), one would expect identical sensitivities to vertical and horizontal orientations because the two stimuli are identical, and therefore indistinguishable.

The open squares represent contrast-sensitivity functions measured with gratings which move in the preferred direction and have the same temporal frequency as their reversing-contrast counterparts. The shape and height of the contrast-sensitivity function obtained by using moving gratings is not significantly different from that measured by using reversing contrast gratings. This finding is consistent with the data of Fig. 3, which indicates that at threshold contrasts, the neuron's response is predominantly direction-insensitive.

The lower pair of contrast-sensitivity functions in Fig. 7 was measured using reversing-contrast gratings, with mean luminance reduced by $3 \log$ units from that used for the upper pair of functions. With vertically-oriented gratings, the peak in the contrast-sensitivity function is less prominent than at $10 \mathrm{~cd} / \mathrm{m}^{2}$, and it occurs at a lower spatial frequency. With hori- (a)

$\uparrow$

Up

(b)

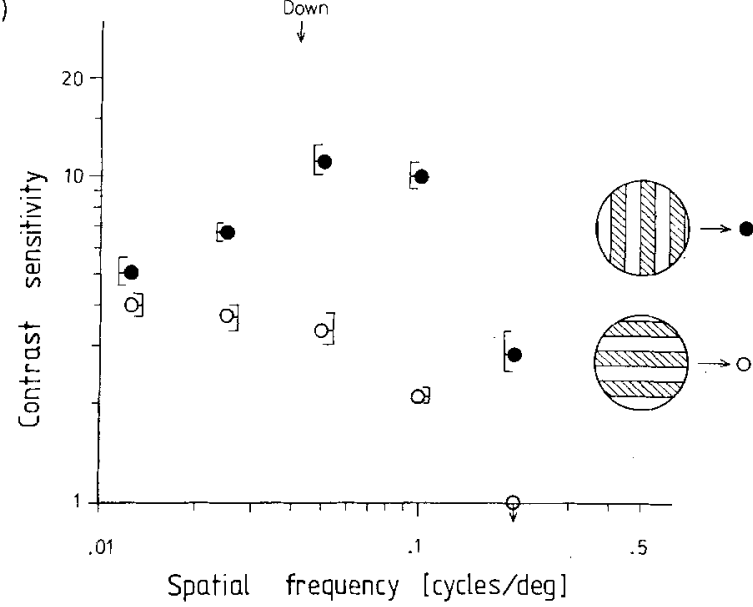

Fig. 8. a Directional selectivity of a lobula-plate neuron with visual field in the contralateral eye and a preferred direction that is mediolateral. Stimulus parameters and response measurement as in Fig. 2. Each data point represents the mean of 4 measurements. Bar: 20 spikes. b Contrast sensitivity functions measured using reversing-contrast gratings oriented along the two directions indicated by the dot-dashed lines in a. Stimulus parameters: $L=10$ $\mathrm{cd} / \mathrm{m}^{2}, f=1.0 \mathrm{~Hz}$. Each data point represents mean \pm s.d. of 4 measurements

zontally-oriented gratings there is no intermediatefrequency peak, as before.

The data of Fig. 7 clearly indicates that visual signals are processed differently along the horizontal and vertical directions, even for stimuli in which there is only flicker, and no movement.

Figures 8 and 9 show data obtained from lobulaplate neurons different from the type with which we routinely worked. The data of Fig. 8 is from a neuron which had its visual field in the contralateral eye and a preferred direction that was medio-lateral (i.e. directed away from the midline), as shown by the polar response plot of Fig. 8 a. Figure $8 \mathrm{~b}$ shows contrastsensitivity functions of this neuron, measured using reversing-contrast gratings oriented along the two directions indicated by the dot-dashed lines. As in the case of the HI DSMD, vertically-oriented gratings yield a contrast-sensitivity function which has peak sensitivity at an intermediate frequency, while horizontally-oriented gratings yield a function that has peak sensitivity at the low-frequency end. Figure 9 shows data from another type of neuron which had more than one preferred direction, as shown by the polar response plot of Fig. 9a. For this neuron, con- 


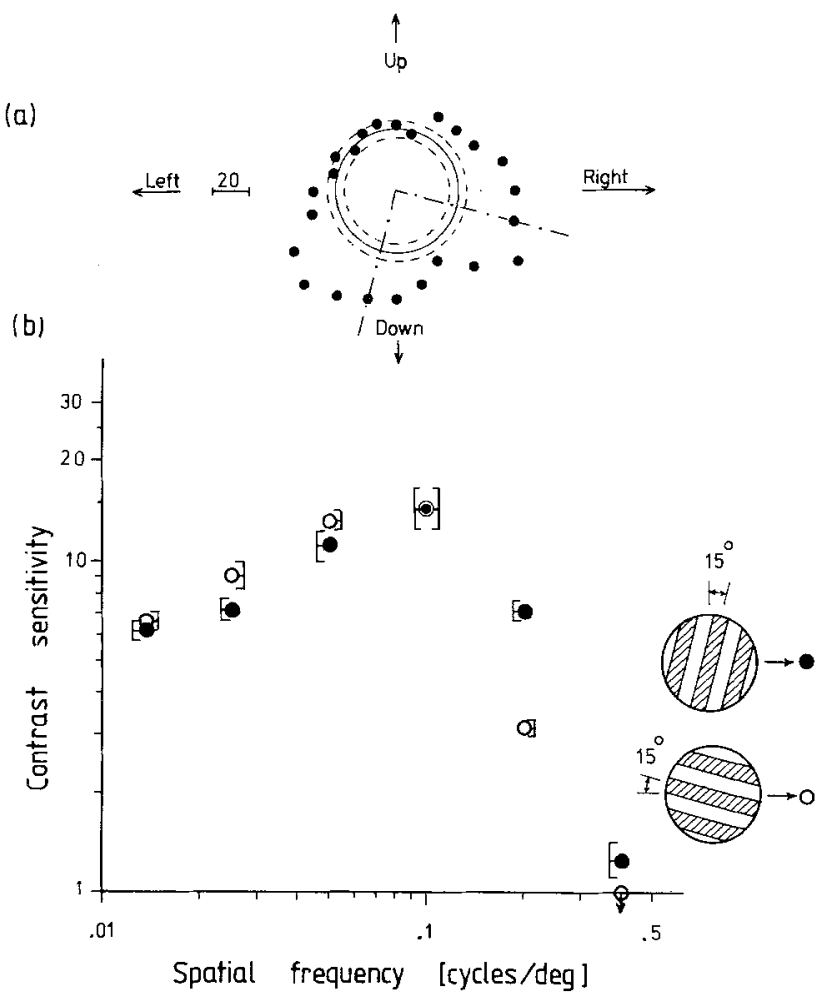

Fig. 9. a Directional selectivity of a lobula-plate neuron which has more than one preferred direction. Stimulus parameters and response measurements as in Fig. 2. Each data point represents the mean of 4 measurements. Bar: 20 spikes. Solid circle and dashed circles on either side indicate spontaneous activity (mean \pm s.d., 20 measurements) which was considerable in this particular neuron. b Contrast sensitivity functions measured using reversingcontrast gratings oriented along the two directions indicated by the dot-dashed lines in a. Stimulus paremeters: $L=10 \mathrm{~cd} / \mathrm{m}^{2}, f=$ $1.0 \mathrm{~Hz}$. Each data point represents mean + s.d. of 4 measurements

trast-sensitivity functions were measured with gratings oriented along two mutually perpendicular directions, as shown by the dot-dashed lines. Both functions had an intermediate-frequency peak (Fig. 9b).

\section{Reversing-Contrast Bars}

Figure 10 shows the response of the HI DSMD to a reversing-contrast bar. The intensity of the bar varies sinusoidally with time, above and below a mean background luminance. The neuron responds primarily to changes of intensity. A burst of impulses closely follows each instant of time at which intensity changes at the maximum rate, regardless of whether it is increasing or decreasing. As in the case of reversingcontrast gratings, there is some habituation.

Figure 11 shows measurements of threshold contrast versus bar width, made with the bar oriented vertically. The temporal frequency of contrast reversals was $1.17 \mathrm{~Hz}$ - almost identical to that used for
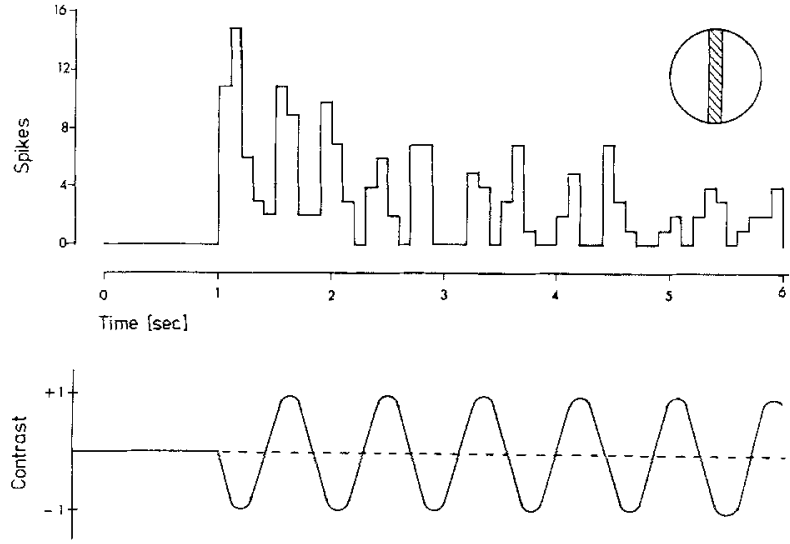

Fig. 10. Histogram of response evoked in HI DSMD by a reversingcontrast bar, accumulated over 4 trials. Stimulus parameters: $L=$ $10 \mathrm{~cd} / \mathrm{m}^{2} ; C=1.0 ; W=4^{\circ} ; f=1.17 \mathrm{~Hz}$. Lower trace shows intensity of bar as a function of time

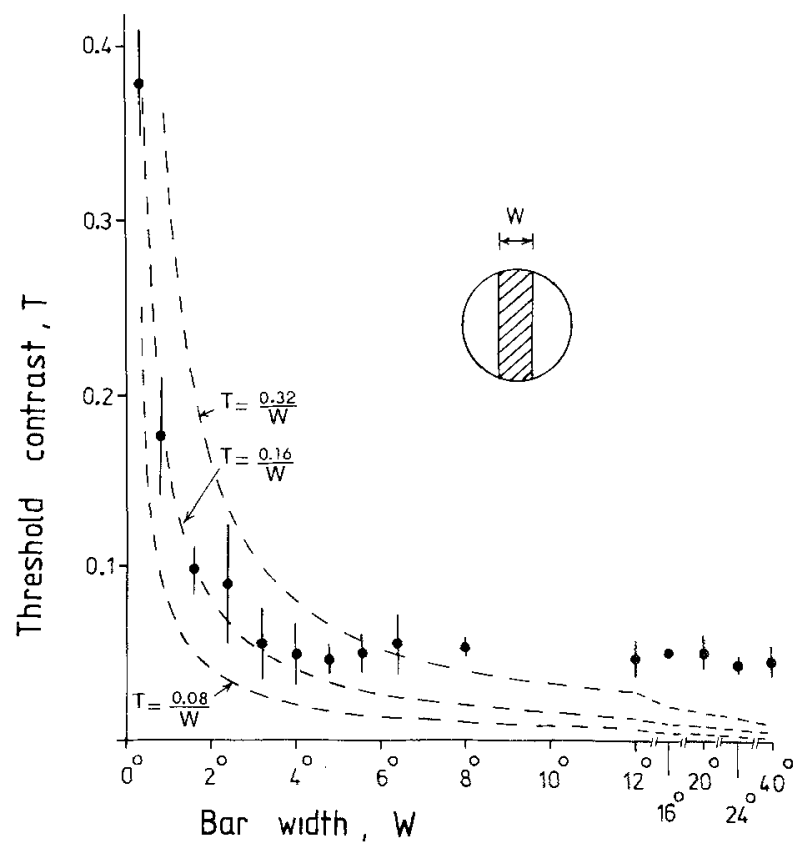

Fig. 11. Threshold contrast $(T)$ versus bar width ( $W$ ), measured for HI DSMD using a vertically-oriented, reversing-contrast bar. Stimulus parameters: $L=10 \mathrm{~cd} / \mathrm{m}^{2}, f=1.17 \mathrm{~Hz}$. Results represent data pooled from 4 animals, with the data from each animal scaled to make $T=0.05$ at $W=16^{\circ}$. Dashed curves represent rectangular hyperbolae

gratings $(1.0 \mathrm{~Hz})$ - and threshold contrast was measured in the same way. For small bar widths $\left(0^{\circ}-2^{\circ}\right)$, threshold contrast $(\mathrm{T})$ is inversely proportional to width (W). Over this range, the data can be adequately approximated by the rectangular hyperbola $T=$ $0.16 / W$. For larger widths $\left(W>4^{\circ}\right)$, threshold contrast is approximately constant, i.e. independent of width. The data is consistent with that expected from a neural array in which linear spatial summation of intensi- 


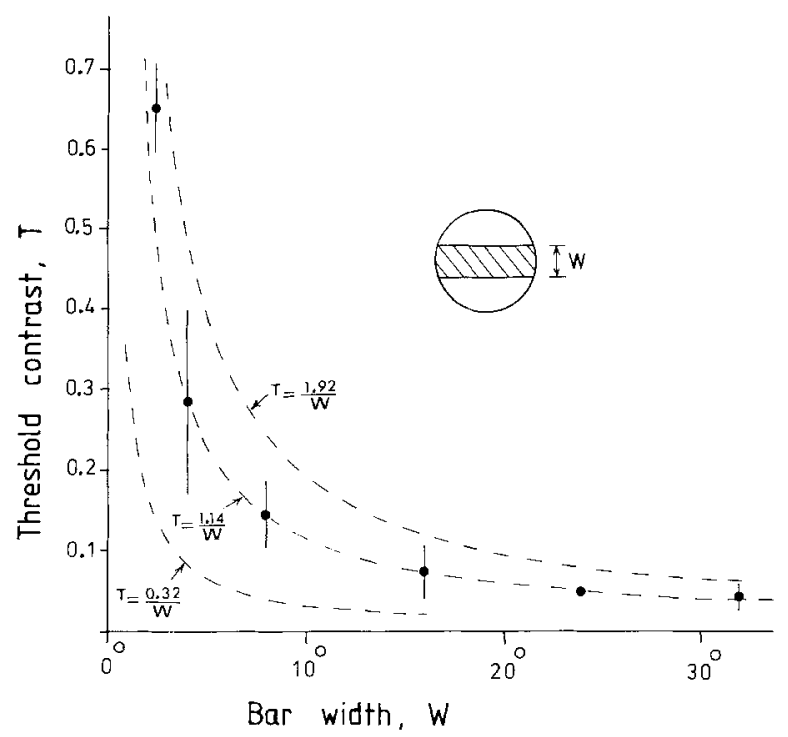

Fig. 12. Threshold contrast $(T)$ versus bar width $(W)$, measured for $\mathrm{Hl}$ DSMD, using a horizontally-oriented, reversing-contrast bar. Stimulus parameters: $L=10 \mathrm{~cd} / \mathrm{m}^{2}, f=1.17 \mathrm{~Hz}$. Results represent data pooled from 5 animals, with the data from each animal scaled to make $T=0.05$ at $W=24^{\circ}$. Dashed curves represent rectangular hyperbolae

ty occurs over an extent of about $2^{\circ}$ in the horizontal direction.

Figure 12 shows measurements of threshold contrast versus bar width, made with the bar oriented horizontally. In this case, threshold contrast is inversely proportional to width for widths ranging from $2^{\circ}$ to as large as $30^{\circ}$. Over the entire range of widths that were tested, the data can be adequately approximated by the rectangular hyperbola $T=1.14 / W$. This finding is consistent with that expected from a neural array in which linear spatial summation of intensity occurs over an extent of at least $30^{\circ}$ in the vertical direction.

\section{Measurements of Crossover Frequency}

When the spatial frequency of a grating exceeds that which can be properly resolved by the visual mosaic of the eye, motion in a given direction can be erroneously interpreted as being in the opposite direction (von Gavel, 1939; Götz, 1965; Zaagman et al., 1977; Eckert, 1980). This condition arises as a result of geometrical interference between the spatial periodicity of the grating and that of the visual mosaic. Here we take advantage of the phenomenon to infer the sampling density of the visual mosaic, and to examine whether the density changes with luminance.

Figure 13 shows the effect of geometrical interference on the response of the Hl DSMD. The visual stimulus is a vertically-oriented, horizontally-moving
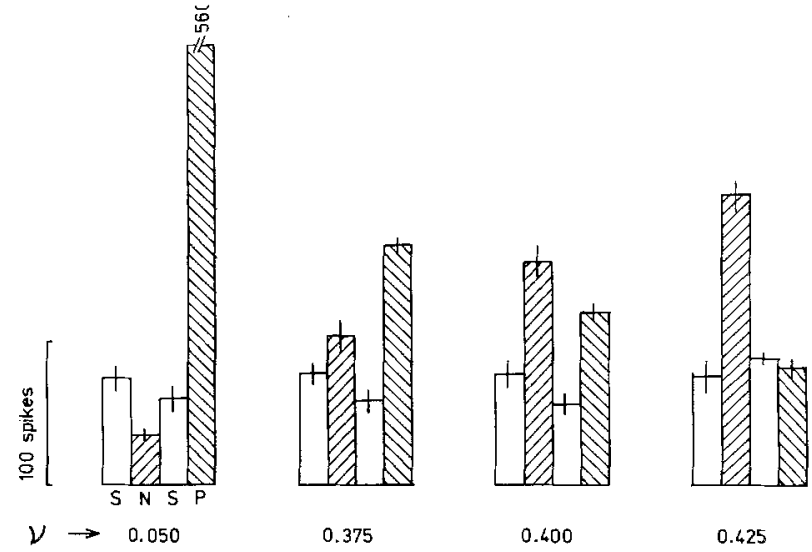

Fig. 13. Measurement of crossover spatial frequency, $v_{c}$. Stimulus is a vertically-oriented grating which undergoes four consecutive 5 -s phases as in Fig. 3. Stimulus parameters: $L=10 \mathrm{~cd} / \mathrm{m}^{2}, C=1.0$, $f=1.17 \mathrm{~Hz}$. Bins show number of spikes in each phase (mean \pm s.d., 4 trials). Number below each set of bins denotes spatial frequency, $v$. For this animal, $v_{c}$ lies between 0.375 cycles/degree and 0.400 cycles/degree

grating. At low spatial frequencies and high contrast, the response evoked by motion in the preferred direction is considerably greater than that evoked by motion in the null direction. As the spatial frequency is increased (adjusting speed to keep temporal frequency constant), the preferred-direction response decreases and the null-direction response increases. The two responses become equal at a spatial frequency between 0.375 and 0.400 cycles/degree. We call this spatial frequency the "crossover frequency". For spatial frequencies higher than the crossover frequency, the null-direction response is greater than the preferred-direction response. That is, the movement detector responds as though it perceives direction of motion erroneously ${ }^{1}$.

1 Note that, while the preferred-direction response is smaller than the null-direction response, it is not suppressed to a level below the spontaneous firing rate, because the direction-sensitive component of the neuron's response is superimposed upon a direction-insensitive component. In an earlier study (Dvorak et al., 1980) we erroneously stated that aliasing (i.e. reversed perception of motion of high-spatial-frequency gratings) does not occur at luminances below $4 \mathrm{~cd} / \mathrm{m}^{2}$. This observation was based on preliminary experiments in which the preferred-direction response was compared with the neuron's spontaneous activity. These experiments did not reveal aliasing because the direction-sensitive component of the neuron's response was being masked by a substantial direction-insensitive component. Under these circumstances, the proper way to test for aliasing is to compare the preferred-direction response with the null-direction response, as we have done here. This procedure isolates the direction-sensitive component of the response by cancelling out the direction-insensitive component 


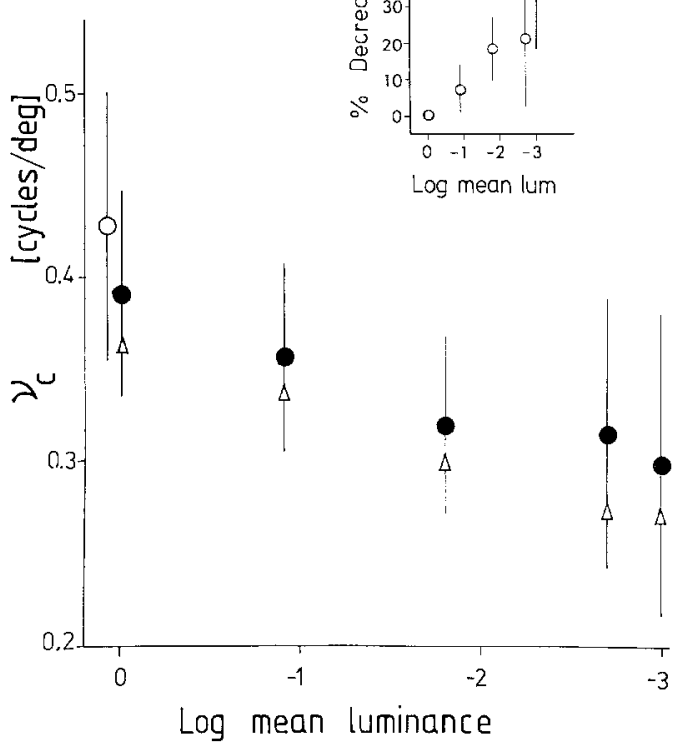

Fig. 14. Crossover spatial frequency, $v_{c}$, versus mean luminance. Zero log mean luminance represents $10 \mathrm{~cd} / \mathrm{m}^{2}$. Filled circles: measurements made using a $53^{\circ}$ stimulus subtense (mean \pm s.d., pooled from 19 animals). Open circle: measurements made using a $28^{\circ}$ stimulus subtense (mean \pm s.d., pooled from 6 animals). Triangles: measurements made on an individual animal using a $28^{\circ}$ stimulus subtense. Inset shows data for the $53^{\circ}$ subtense, re-plotted as percentage decrease in crossover frequency relative to the value at $10 \mathrm{~cd} / \mathrm{m}^{2}$

At high luminance $\left(10 \mathrm{~cd} / \mathrm{m}^{2}\right)$, crossover frequencies can typically be measured with a precision of \pm 0.0125 cycles/degree, which implies that the precision of the measurement is limited only by the spatial-frequency resolution of our stimulus generating apparatus. At low luminances, precision deteriorates to \pm 0.025 cycles $/$ degree, due to poorer signal-to-noise ratio in the response.

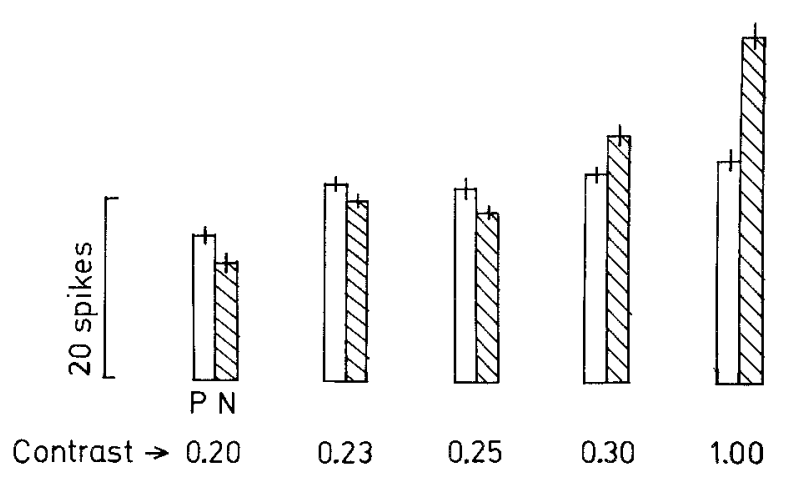

Fig. 15. Variation of crossover spatial frequency with contrast Stimulus is a vertically oriented grating which undergoes 4 consecutive 5-s phases, as in Fig. 3. Stimulus parameters: $L=10 \mathrm{~cd} / \mathrm{m}^{2}$, $v=0.425$ cycles/degree, $f=11.7 \mathrm{~Hz}$. Bins show number of spikes (mean \pm s.d., 4 trials) that occur during motion in preferred $(P)$ and null $(N)$ directions, for various contrasts

Figure 14 shows crossover frequency measured as a function of luminance. At the highest luminance $\left(10 \mathrm{~cd} / \mathrm{m}^{2}\right)$, the mean crossover frequency is $0.39 \mathrm{cy}-$ cles/degree, when measured with high-contrast gratings. As luminance is lowered, the mean crossover frequency progressively decreases until it is $0.30 \mathrm{cy}$ cles/degree when the luminance is reduced by $3 \log$ units. The variability of the data is partly due to differences in crossover frequency that exist among individual animals at any given level of luminance. Thus, the variability in the measurements at low luminances is reduced when the data is plotted in terms of percentage reduction of crossover frequency, relative to the value at high luminance (see inset of Fig. 14). When luminance is reduced by $3 \log$ units, the mean crossover frequency decreases by about $30 \%$. This luminance-dependent variation persists when the angular subtense of the stimulus is reduced from the usual $53^{\circ}$ to $28^{\circ}$ (open triangles, Fig. 14).

Table 1

\begin{tabular}{|c|c|c|c|c|c|c|}
\hline \multirow[t]{2}{*}{ Unit \# } & \multirow[t]{2}{*}{$\begin{array}{l}\text { Stimulus } \\
\text { subtense }\end{array}$} & \multirow{2}{*}{$\begin{array}{l}\text { Temporal } \\
\text { freq. } \\
(\mathrm{Hz})\end{array}$} & \multicolumn{2}{|c|}{$\begin{array}{l}\text { Crossover spatial freq. } \\
\text { (cycles/deg) }\end{array}$} & \multirow[t]{2}{*}{$\begin{array}{l}\% \\
\text { Decrease }\end{array}$} & \multirow{2}{*}{$\begin{array}{l}\text { Precision of } \\
\text { measurement } \\
\text { (Cycles/deg) }\end{array}$} \\
\hline & & & Low contrast & High contrast & & \\
\hline \multirow{3}{*}{790501} & $53^{\circ}$ & 0.117 & 0.4375 & 0.4125 & 5.7 & \pm 0.0125 \\
\hline & $53^{\circ}$ & 1.17 & 0.4125 & 0.4000 & 3.0 & \pm 0.0125 \\
\hline & $53^{\circ}$ & 11.7 & 0.4250 & 0.4000 & 5.9 & \pm 0.0125 \\
\hline 790404 & $53^{\circ}$ & 1.17 & 0.3625 & 0.3125 & 13.8 & \pm 0.0125 \\
\hline 790207 & $53^{\circ}$ & 1.17 & 0.4375 & 0.3875 & 11.4 & \pm 0.0125 \\
\hline \multirow{3}{*}{790406} & $28^{\circ}$ & 0.117 & 0.5000 & 0.5000 & 0.0 & \pm 0.0125 \\
\hline & $28^{\circ}$ & 1.17 & 0.4875 & 0.4875 & 0.0 & \pm 0.0125 \\
\hline & $28^{\circ}$ & 11.7 & 0.5125 & 0.4875 & 4.9 & \pm 0.0125 \\
\hline 790405 & $28^{\circ}$ & 1.17 & 0.4875 & 0.4625 & 5.1 & \pm 0.0125 \\
\hline
\end{tabular}


Crossover frequency varies continuously with luminance and not in a stepwise fashion, at least within the limits of resolution of our data.

Crossover frequencies are slightly higher when measured with low-contrast gratings rather than highcontrast gratings. Figure 15 illustrates this by showing how the responses to motion in the preferred and null directions change as the contrast of a $0.425 \mathrm{cy}-$ cles/degree grating is increased from low to high values. For contrasts below 0.25 , the preferred-direction response is greater than the null-direction response, indicating that the crossover frequency is greater than 0.425 cycles/degree. For contrasts above 0.25 the opposite is true, indicating that the crossover frequency is now smaller than 0.425 cycles/degree. The variation of crossover frequency with contrast is small but measurable (Table 1), and it tends to be smaller when the angular subtense of the stimulus is reduced from the usual $53^{\circ}$ to $28^{\circ}$. The implications of these findings will be discussed in a subsequent section.

\section{Discussion}

It is fruitful to interpret the results in the context of what is already known regarding the anatomy and physiology of the visual pathway of the fly. Accordingly, we begin this discussion with a brief review.

The most striking anatomical feature of the visual pathway of the fly, and of many other insects, is the extraordinary spatial regularity and homogeneity of the various visual ganglia (revs. Reichardt, 1970; Braitenberg, 1972; Strausfeld, 1976; Kirschfeld, 1978). The retinula cell axons project to the first optic ganglion, the lamina, in an orderly and spatially-repetitive fashion, synapsing with the second-order neurons within neural units known as cartridges. The array of ommatidia on the surface of the compound eye bears a one-to-one correspondence with the array of cartridges in the lamina. The lamina cartridges in turn project to as many neural units, known as columns, in the second optic ganglion, the medulla. Thus the retinotopic projection is preserved at least up to the level of the medulla, and probably extends even beyond it into the lobula and lobula plate. However, at each stage of the visual pathway, information is filtered, transformed, or otherwise analyzed (McCann, 1974; Zettler and Weiler, 1976; Laughlin and Hardie, 1978; Shaw, 1978).

Direction of motion is determined by comparing, or correlating, signals from "sampling units" which have small receptive fields, and which look at neighbouring regions of visual space (rev. Reichardt, 1969). It is not yet known precisely where along the visual pathway this correlation occurs. It certainly does not

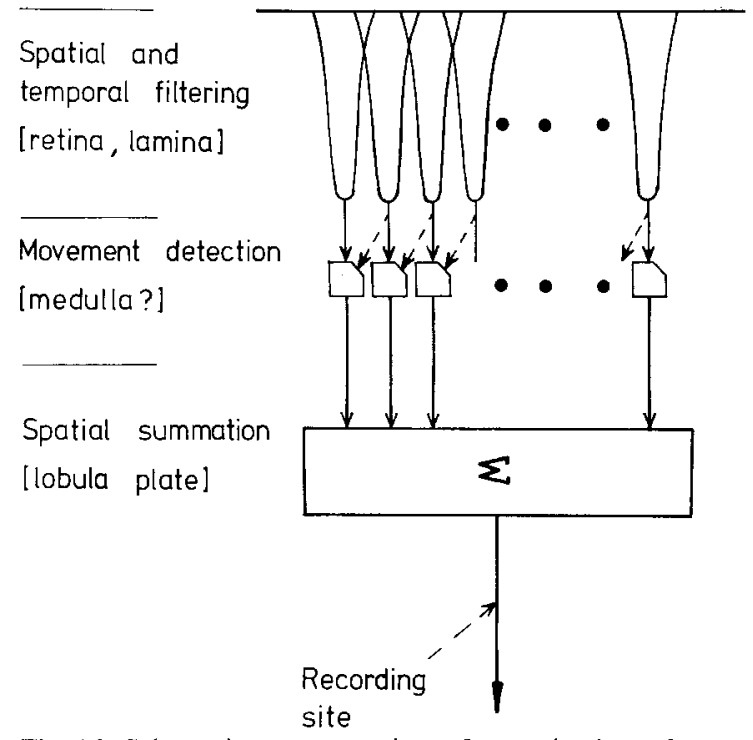

Fig. 16. Schematic representation of organization of movementdetecting pathway. Description in text

take place in the retina, and to this date, there is no evidence of its occurring in the lamina. Recent experiments with the activity-specific stain deoxyglucose point to the medulla (Buchner et al., 1979), and direction-selective units have been found therein (DeVoe and Ockleford, 1976). However, the actual site is not important for our interpretation.

The DSMD neurons of the lobula plate, generally speaking, have dendritic arborizations which extend over large portions of the visual mosaic (Dvorak et al., 1975; Pierantoni, 1976; Hausen, 1976; Eckert and Bishop, 1978). In functional terms, their responses reflect spatial summation of the responses of a large array of "elementary" movement detectors (Bishop et al., 1968; Marmarelis and McCann, 1973).

The analysis of visual information by the movement-detecting pathway can be considered to take place in three successive stages: (i) peripheral filtering of visual signals in space and time, and modification of amplitude, (ii) elementary movement detection and (iii) spatial summation of the responses of elementary movement detectors. These operations and their presumed locations are schematically depicted in Fig. 16.

\section{Reversing-Contrast Stimuli}

While the obvious and most powerful stimulus to a DSMD neuron is a visual pattern moving in the preferred direction, reversing-contrast patterns possess two special advantages. Firstly, moving patterns are not suitable for measuring contrast-sensitivity functions along the direction perpendicular to the preferred-null axis, because motion along this direction 
evokes responses that are weak and qualitatively different from those evoked by motion parallel to the preferred-null axis. Reversing-contrast gratings are immune to this problem; they can be used to measure contrast-sensitivity functions along any direction, because they possess no movement component (or equivalently, equal and oppositely directed components).

Figures 4 and 10 highlight three features of the DSMD neuron's response to reversing-contrast stimuli. Firstly, the neuron's response is phasic. That is, responses are evoked only by changing patterns and not by static ones. Secondly, the response shows some habituation. Thirdly, changes of intensity are encoded in a nonlinear fashion. Both increase and decrease of intensity are signalled by a transient increase in firing rate (Fig. 10), this rectifying action producing the burst of impulses that occurs at each reversal of the grating (Fig. 4). The rectification must arise at the level of the sampling units or at the level of the elementary movement detectors. If the rectification occurred at a level subsequent to spatial summation (e.g. the level at which the DSMD encodes its response), and if all the neuronal circuitry preceding this level operated in a linear fashion, then it is difficult to imagine how a neuron with linear spatial summation over the entire eye (nearly $180^{\circ}$ ) could respond to gratings of spatial frequency as high as 0.5 cycles/ degree.

\section{Interpretation of Contrast-Sensitivity Function}

In general, one would expect the shape of the contrast-sensitivity function to depend not only upon peripheral filtering, but also upon the processes of elementary movement detection (Buchner, 1976) and subsequent spatial summation. However, we shall argue below that under our experimental conditions, the measured contrast-sensitivity function primarily reflects the spatial filtering that precedes movement detection.

Directional selectivity of the DSMD is poor at low contrasts; under threshold conditions, the response to a moving grating is dominated by the direction-insensitive component (Fig. 3). This suggests that for the conditions under which the contrast-sensitivity function is measured, the lateral interactions that mediate directional selectivity (shown by dashed lines in Fig. 16) are inactive, or only weakly active. The idea is reinforced by the data of Fig. 7, which shows that the contrast-sensitivity function measured by using gratings moving in the preferred direction (open squares) is not significantly different from that measured by using reversing-contrast gratings with the same temporal frequency. In the light of these consid- erations, we propose that the contrast-sensitivity functions measured with reversing-contrast gratings reflect spatial processing that occurs peripheral to the neuronal site at which directionally-selective movement detection takes place.

In interpreting the contrast-sensitivity function data, we make the following assumptions: (i) The DSMD produces a criterion response when the total response of the array of sampling units, spatially summed over the visual field of the DSMD, equals a fixed value. (ii) All sampling units have identical receptive fields. (iii) Each sampling unit integrates spatial variations of intensity linearly, with a weighting function specified by its receptive-field profile. The response of the sampling unit need not be encoded in a linear fashion; for example, it can be a rectified version of a linear response. Under the above conditions, the inverse Fourier transform of the DSMD's contrast-sensitivity function would infer the receptive-field profile of a sampling unit.

Consider first the contrast-sensitivity functions measured at the highest luminance (Fig. 7). Along the horizontal plane, sensitivity peaks at an intermediate frequency, predicting a receptive field profile with an excitatory centre and inhibitory flanks. The falloff in sensitivity at the high-frequency end specifies the shape and width of the excitatory centre, while the falloff at the low-frequency end specifies the shape and width of the inhibitory flanks (Enroth-Cugell and Robson, 1966). Along the vertical plane, sensitivity is maximum at the low-frequency end and it falls off toward high frequencies, predicting a receptivefield profile that is purely excitatory.

By calculating the inverse Fourier transform of the contrast-sensitivity functions, we have inferred the receptive-field profile of a sampling unit in two dimensions. The results are shown in Fig. $17 \mathrm{a}, \mathrm{b}$, and details of the calculation are in Appendix A. At high luminance $\left(10 \mathrm{~cd} / \mathrm{m}^{2}\right)$, the inferred receptive-field profile has an excitatory centre with a half-width of $2^{\circ}$ along the horizontal direction and $2.2^{\circ}$ along the vertical direction, and diffuse inhibitory flanks that extend only along the horizontal direction (Fig. 17a). At the lower luminance $\left(10^{-2} \mathrm{~cd} / \mathrm{m}^{2}\right)$, the contrast-sensitivity function along the vertical direction is essentially unchanged. The contrast-sensitivity function along the horizontal direction falls off more rapidly at the high-frequency end and its peak shifts toward lower frequencies. The inferred receptive-field profile has an excitatory centre which is wider along the horizontal direction (half-width $=3.1^{\circ}$ ) and inhibitory flanks that are weaker and more diffuse (Fig. 17a), compared to those at high luminance.

That the falloff in sensitivity at low spatial frequencies is indeed due to lateral inhibition, is sup- 

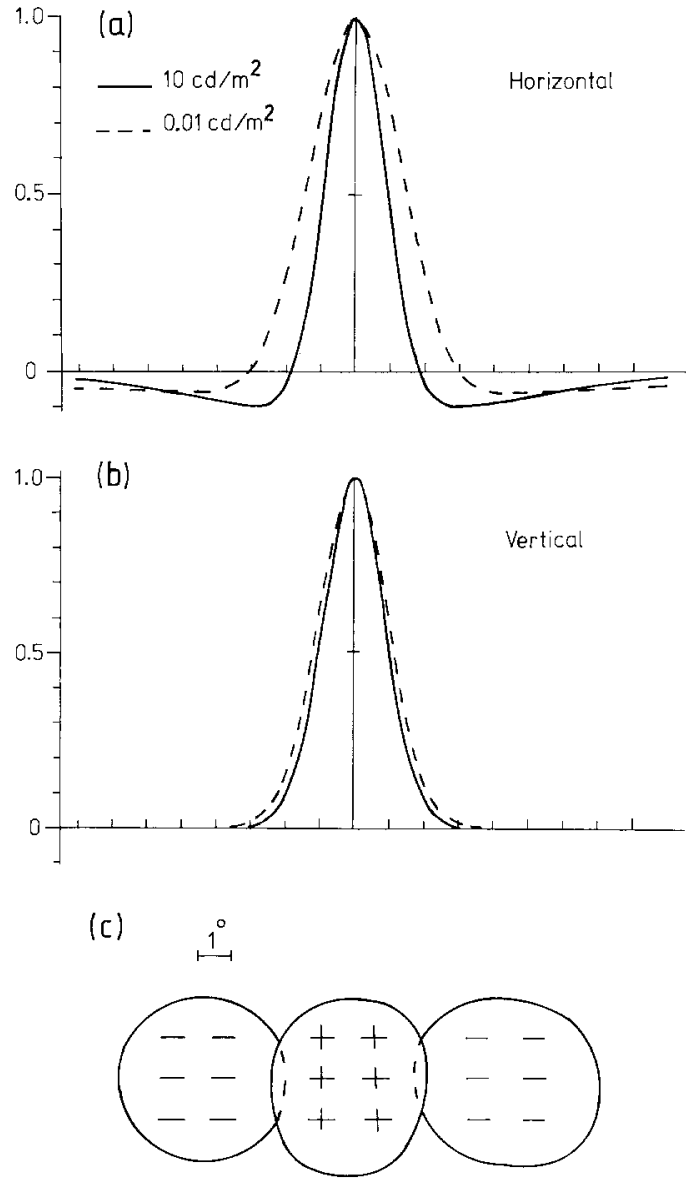

Fig. 17a-c. Receptive-field profile of a sampling unit along a the horizontal plane and $\mathbf{b}$ the vertical plane. These profiles were inferred from the contrast-sensitivity data of Fig. 7 by inverse Fourier transformation, as described in the Discussion and Appendix A. Each division of the abscissa in $\mathbf{a}$ and $\mathbf{b}$ represents $1^{\circ}$. $\mathbf{c}$ shows the excitatory and inhibitory zones of a "sustaining unit" in the chiasma, as reported by Arnett (1972); redrawn from Fig. 3 of that paper, with the author's permission. Scale of $\mathbf{c}$ matches those of $\mathbf{a}$ and $\mathbf{b}$

ported by control experiments which rule out alternative interpretations. The shape of the measured contrast-sensitivity function is not altered when the angular subtense of the stimulus is increased by a factor of two, indicating that the low-frequency attenuation is not an artifact created by the finite angular extent of the stimulus. Furthermore, the low-frequency attenuation disappears when the contrast-sensitivity function is measured at low luminance. It also disappears when the contrast-sensitivity function is measured at high temporal frequencies, as it should if it were due to lateral inhibition. Details of these and other control experiments are in Dvorak et al. (1980).

In the vertebrate visual system, it is believed that directional selectivity is mediated by inhibitory interactions occurring between sampling units (Barlow and
Levick, 1965). Is the inhibition inferred by our experiments a reflection of these interactions? We think not, because (a) the low-frequency attenuation is present even at low (threshold) contrasts, when directional selectivity is weak or absent; (b) the spatial extent of the inferred inhibition is large compared to the distance over which the movement-detecting system compares signals to determine direction of movement (see section on crossover experiments). For these reasons, we believe that the inferred lateral inhibition occurs at a site that is peripheral to that at which directionality is engendered. In other words, the inferred inhibition must be an integral part of the receptive fields of the sampling units.

\section{Interpretation of Bar Experiments}

We have interpreted the results of the experiments with the reversing-contrast gratings in terms of the HI DSMD receiving and summing responses from an array of sampling units, each unit having a receptive field profile with an excitatory centre and inhibitory flanks, as in Fig. 17 a, b. We shall show below that the results of the bar experiments are consistent with this interpretation.

Consider the experiment with the horizontal bar (Fig. 12). The data is well approximated by the rectangular hyperbola $T=1.14 / W$, indicating that threshold contrast, $T$, is inversely proportional to bar width, $W$, over a large range of widths $\left(2^{\circ}-30^{\circ}\right)$. This finding is consistent with each sampling unit having no inhibitory flanks along the vertical direction. In such a situation, the vertical distance over which signals are spatially summed would be limited only by the size of the receptive field of the DSMD as a whole, and not by the size of individual sampling units.

The experiment with the vertical bar, on the other hand, yields qualitatively different results. It is clear from Fig. 11 that the data of threshold contrast versus bar width cannot be approximated by a single rectangular hyperbola over the entire range of bar widths. This finding is consistent with each sampling unit having a receptive field that consists of an excitatory centre, and inhibitory flanks extending along the horizontal direction. In such a situation, spatial summation would be effective only over horizontal distances that are smaller than the width of the excitatory centre of a sampling unit. For bar widths less than $2^{\circ}$, the data is well approximated by the rectangular hyperbola $T=0.16 / W$. This finding is consistent with the horizontal half-width of $2^{\circ}$ that was inferred for the excitatory centre of a sampling unit, using reversing-contrast gratings (see Fig. 17a). When the bar is much 
wider than the excitatory centre of a sampling unit, then a unit which is located well within the boundary of the bar would produce a weaker response than a unit which is positioned so that its excitatory centre lies just within the boundary. Although both units would receive equal excitatory inputs from their receptive-field centres, the former unit would receive inhibitory signals from both inhibitory flanks while the latter unit would receive inhibitory signals only from one flank, namely that covered by the bar. Thus, for large bar widths, threshold contrast should be independent of width because threshold responses would be elicited only by the boundaries of the bar. This expectation is borne out by the results, which indicate that threshold contrast for a vertical bar is constant for widths greater than $4^{\circ}$. Thus in terms of our model, the results of the bar experiments are consistent with those of the grating experiments.

\section{Sustaining Units in the Chiasma}

The receptive field profiles which we have inferred for the sampling units are remarkably similar to those reported by Arnett (1972) for "sustaining units" recorded from the intermediate chiasma which connects the lamina to the medulla. Arnett's study was on North American Phaenicia sericata, which is taxonomically identical to the Australian sheep blowfly $\mathrm{Lu}$ cilia sericata used in our study (pers. comm. from K.R. Norris to S. Shaw). Figure $17 \mathrm{c}$, redrawn from Fig. 3 of Arnett's paper, illustrates the excitatory and inhibitory zones of the receptive field of a sustaining unit. According to Arnett's measurements of angular sensitivity, these units have an excitatory centre with a half-width of $2.2^{\circ}$ measured along the horizontal direction and $2.6^{\circ}$ measured along the vertical direction, and inhibitory flanks that extend only in the horizontal direction out to about $5^{\circ}$ on either side of the unit's visual axis. Within the limits of experimental error, these figures agree very well with the angular-sensitivity profiles that we have inferred; compare Fig. 17c with Fig. 17a,b. Our findings predict that the inhibitory flanks are weak and diffuse compared to the excitatory centre, and that sensitivity to a spot of light is at least 10 times greater at the centre of the excitatory region than at the inhibitory zones (Fig. 17b). This prediction parallels Arnett's observation that sensitivity, measured with a spot of light, is $1.3 \log$ units higher in magnitude at the centre of the excitatory region than at the (presumably) most effective location within the inhibitory region. (Note, however, that the inhibition can have a substantial effect when the visual stimulus is not a small spot, but a wide bar. This is because the inhibitory flanks cover a much larger area than the excitatory centre). All of the above similarities, plus the observation that the sustaining units and the DSMD have similar spectral sensitivities (McCann and Arnett, 1972) make Arnett's sustaining units promising candidates for the sampling units in the neural pathway that links the retina to the H1 DSMD. Evidence of such a role for the sustaining units was first presented by McCann (1973), and our data provides additional support for this notion. Conclusive proof, however, is not yet available and the participation of other types of lamina units - such as the "on-off" units (Arnett, 1972), the units studied by Mimura (1974) or the spiny monopolar neurons L1 through L3 (rev. Zettler and Järvilehto, 1972; Laughlin, 1980)- cannot be ruled out at the present time.

Regardless of whether or not Arnett's sustaining units are indeed involved, it would appear that sampling units with receptive fields consisting of an excitatory centre and horizontally-extending inhibitory flanks - as inferred by our study - are ideal for filtering the signals that drive a horizontal movement detector. Such a receptive-field organization would emphasize spatial variations of intensity in the horizontal direction, enhancing sensitivity to the horizontal component of movement. At the same time, it would attenuate spatial variations of intensity along the vertical direction, which are of no use in detecting horizontal movement. Given the restricted amplitude range of any signalling device - in particular, the sampling units (see, for example, Laughlin and Hardie, 1978) - the available range would be utilized most efficiently if the neuron carries only useful information, in this case information pertaining to horizontal movement. Our findings suggest that the neuronal circuitry which achieves this spatial "tuning", or filtering, is located distal to the site at which directionally-selective movement detection occurs, possibly in the lamina. Spatial prefiltering of visual signals is examined in greater detail under "Functional Significance".

\section{Interpretation of Crossover Experiments}

Any mechanism that exhibits directional selectivity must compare or correlate information from at least two distinct regions of the visual scene. The object of the crossover experiments was to measure how far apart these regions are, and to examine whether their separation changes with luminance.

Consider a movement detector which discriminates leftward movement from rightward movement by correlating responses from sampling units with visual axes azimuthally separated by an angle $\widetilde{A \phi}$. Such a detector will correctly determine the 
direction of motion of a vertically-oriented, horizontally-moving grating if the spatial frequency $v$ of the grating is lower than $\frac{1}{2 \widetilde{\Delta \phi}}$. If the spatial frequency is higher than this limit and in the range $\frac{1}{2 \widetilde{\pi \phi}}<v<\frac{1}{\widetilde{\Delta \phi}}$, then motion in a given direction will be erroneously interpreted as being in the opposite direction, because the spacing of the sampling units is not close enough to resolve spatial frequencies in this range (Götz, 1965; Buchner, 1976; Zaagman et al., 1977; Eckert, 1980).

Figure 13 illustrates the phenomenon. For spatial frequencies lower than about 0.4 cycles/degree, the preferred-direction response is greater than the nulldirection response; for frequencies higher than 0.4 cycles/degree, the opposite is true. The inferred value of $\widetilde{\Delta \phi}$ is therefore $\frac{1}{(2 \times 0.4)}=1.25^{\circ}$. The figure agrees reasonably well with the interommatidial angle measured between adjacent ommatidia of a horizontal row, in the frontal eye region $\left(1.2^{\circ}\right.$; Ribi and Dvorak, unpublished measurements). This correspondence between the inferred $\widetilde{\Delta \phi}$ and the ommatidial geometry is in accord with the findings of Zaagman et al. (1977).

When the mean luminance is lowered by $3 \log$ units from the maximum level $\left(10 \mathrm{~cd} / \mathrm{m}^{2}\right)$, the inferred $\widetilde{\Delta \phi}$ increases from about $1.3^{\circ}$ to $1.7^{\circ}$ (Fig. 18). The experiment with the $23^{\circ}$ aperture (instead of the usual $53^{\circ}$ aperture) demonstrates that variations of $\widetilde{\Delta \phi}$ with luminance occur even within restricted regions of the eye (open triangles, Fig. 14). Therefore, the luminance-dependent variations of $\widetilde{\Delta \phi}$ that are measured at the larger stimulus subtense are not due to different eye regions (with different $\widetilde{\Delta \phi}$ 's) being functional at different luminances.

Note that $\widetilde{\Delta \phi}$ denotes the separation of a pair of correlating sampling-units that would have a crossover spatial frequency equal to that measured. This quantity is an "effective" separation, which in reality may reflect the net effect of more than one pair of correlating sampling-units, each pair having a different separation (Buchner, 1976; Pick and Buchner, 1979; also, see below). Our experiments cannot resolve this question. At each luminance, we measure the smallest spatial frequency at which crossover occurs (i.e. preferred-direction response equals null-direction response). In theory, additional crossovers should occur at multiples of this frequency, but these are difficult to locate because the response becomes weaker as the spatial frequency of the grating is increased.

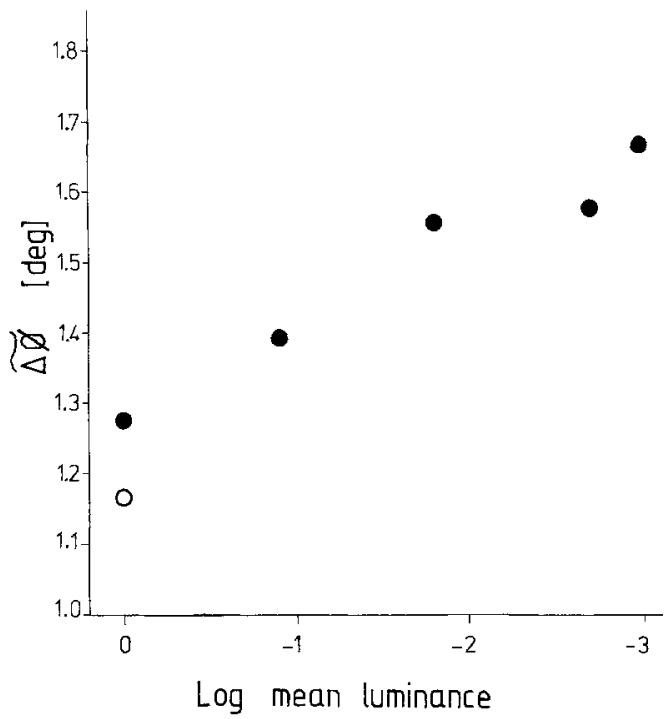

Fig. 18. Variation of $\widetilde{\Delta \phi}$ with luminance. The values of $\widetilde{\Delta \phi}$ are inferred from the measurements of crossover frequency (Fig. 14) (see text). Zero $\log$ mean luminance: $10 \mathrm{~cd} / \mathrm{m}^{2}$. Filled circles: inferred mean values of $\widetilde{\Delta \phi}$ for the $53^{\circ}$ stimulus subtense. Open circle: inferred mean value of $\widetilde{A \phi}$ for the $28^{\circ}$ stimulus subtense

\section{Possible Neural Mechanisms}

Our interpretation of the results of this paper can be summarized as follows. The H1 DSMD receives input via sampling units that have receptive fields comprising an excitatory centre, and inhibitory flanks extending along the horizontal direction. As luminance is lowered the excitatory centre widens, the inhibitory flanks become weaker and more diffuse, and the effective separation between the visual axes of correlating sampling units increases. In other words, the functional "mosaic" of the eye becomes coarser.

While the manuscript of this paper was being reviewed, Pick and Buchner (1979) published a related study on visual movement detection under light and dark adaptation in the fly. They measured behavioural turning responses evoked by stimuli consisting of moving gratings, or a pair of flickering bars. The present study confirms theirs in pointing to lateral inhibition at the periphery of the movementdetecting pathway and to a coarsening of the visual mosaic as luminance is lowered. According to Pick and Buchner, the luminance-dependent changes that are observed could be interpreted either in terms of increased pooling of peripheral signals, or, alternatively, in terms of extended movement-specific interactions (i.e., increased $\widetilde{\Delta \phi}$ ). Our experimental approach eliminates this ambiguity and measures each effect separately by taking advantage of the observation that the movement-specific interactions are 
a

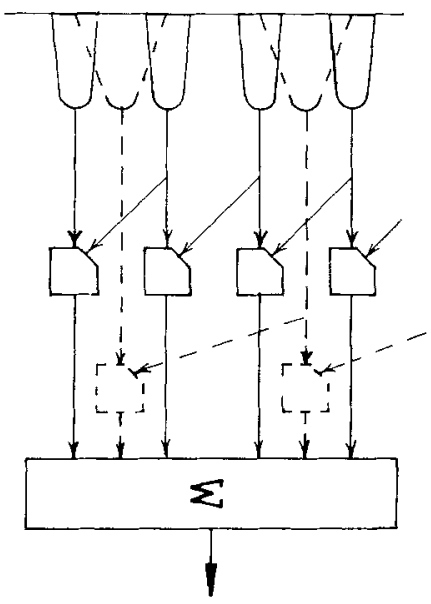

b

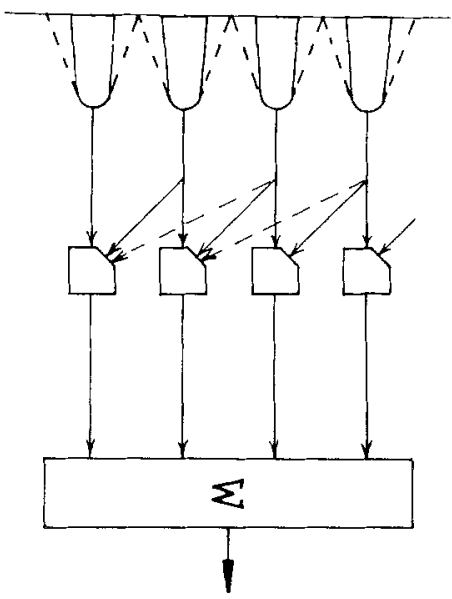

c

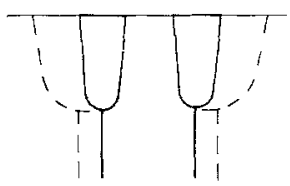

Fig. 19a-c. Three neuronal models that can account for the observed luminance-dependent changes in the characteristics of the movement detector. Description in text weak at threshold contrast. Thus, we infer peripheral pooling by measuring the contrast sensitivity function, and unravel movement-specific interactions by measuring the crossover spatial frequency.

How might the luminance-dependent changes of the movement-detecting pathway be mediated in terms of neuronal circuitry? There are several possible ways, three of which are illustrated in Fig. 19.

In model $a$, there is more than one array of elementary movement detectors and the response of the DSMD is a weighted sum of the outputs of the arrays. For simplicity, let us consider two arrays. One array is a high-acuity array, receiving input from sampling units which are closely spaced, and have relatively narrow receptive fields. The other is a lowacuity array, receiving input from sampling units which are spaced further apart, and have broader receptive fields. The response of the DSMD is a weighted sum of the responses of the two arrays. At high luminance, the relative weighting of the two responses is such that the high-acuity array is favoured, so that its characteristics dominate the response. As luminance is decreased, the weighting gradually changes so that eventually the low-acuity array "takes over", just like the rod system takes over from the cone system in human vision (Wyszecki and Stiles, 1967). There cannot be an abrupt transition from one array to the other, since the experimentally-measured crossover frequency varies continuously with luminance.

Model $a$ offers a testable prediction. If it holds, then it might be possible to isolate the response of the high-acuity array by using a moving grating of high spatial frequency and adjusting its contrast so that the stimulus is suprathreshold for the high-acuity array, but subthreshold for the low-acuity array. The crossover frequency measured under such conditions should yield $\widetilde{A \phi}$ corresponding to the high-acuity array. If the contrast is now increased to a level such that the low-acuity array is also above threshold, the crossover frequency should decrease to a value in between those corresponding to the high acuity and low acuity arrays, because the DSMD's response now reflects the characteristics of both arrays.

The data of Fig. 15 indicates that the measured crossover frequency does depend on contrast. Table 1 shows example values of crossover frequency measured at high luminance, using gratings of low and high contrast. Although the values are consistently lower when measured with high-contrast gratings as predicted by the model - the differences are too small to account for the extent to which the crossover frequency decreases when luminance is lowered $(30 \%$; see inset of Fig. 14).

We feel that the small differences shown in Table 1 arise from the geometry of the stimulus and inhomogeneity of the eye, rather than from two (or more) parallel arrays of sampling units. For a grating that subtends a visual angle of $53^{\circ}$, the (angular) spatial frequency, as seen by the fly, increases by $25 \%$ in going from the centre to the periphery (see "Methods"). Sampling units that view the centre of the grating see the lowest spatial frequency; consequently, they would have the lowest contrast threshold, assuming that the eye is homogeneous over the region that views the grating. Therefore at low contrasts, the measured crossover frequency would reflect the performance of the region of the eye that views the centre of the grating. At high contrasts, the peripheral regions would also exceed threshold, and their contributions would be recruited into the DSMD's response. The measured crossover frequency would now be lower because the peripheral regions see a higher spatial frequency than the central region. If inhomogeneity of the eye is taken into account even larger variations of crossover frequency with contrast can be expected, because acceptance angles of retinula cells are usually narrowest (Hardie, 1978) and interommatidial angles smallest (e.g. Collett and Land, 1975; Beersma et al., 1975) in the frontal region of the eye. As expected from these considerations, higher crossover frequencies (open circle, Fig. 14) and smaller variations of crossover frequency 
with contrast (units 790405 and 790406 , Table 1) are obtained when the stimulus subtense is reduced to $28^{\circ}$. In summary, the small variations of crossover frequency with contrast can be entirely accounted for on the basis of stimulus geometry and inhomogeneity of the eye.

We conclude that, if there is more than one array in the movement-detecting pathway, it is not possible to isolate the response of an individual array by manipulating contrast. Table 1 also shows that it is not possible to isolate the response of an individual array by manipulating the temporal properties of the stimulus. Within the range of grating speeds that our apparatus can generate, crossover frequency is essentially independent of speed. Compare the crossover frequencies shown in Table 1 (units 790501 and 790406) for temporal frequencies of $0.117 \mathrm{~Hz}, 1.17$ $\mathrm{Hz}$ and $11.7 \mathrm{~Hz}$. Thus, the available experimental evidence argues against model $a$.

In model $b$, there is only one array of sampling units and elementary movement detectors. As luminance is decreased, each sampling unit widens its excitatory centre by increasing the spatial pool of receptors that contribute to it. The movement-detecting process is simultaneously modified to use signals not only from neighbouring sampling units, but also from units that are more remote.

Model $c$ - illustrated for just one elementary movement detector, for clarity - simultaneously achieves widening of excitatory centres and increase of $\widetilde{\Delta \phi}$ by modifying receptive-field profiles in an asymmetrical fashion, as shown by the dashed lines. Due to the asymmetry that is inherent to this model, a given sampling unit cannot be shared by neighbouring elementary movement detectors, so there can be only half as many elementary movement detectors as sampling units.

As an example of model $c$, consider a pair of correlating sampling units receiving signals from an array of 4 retinular cells $r_{1}, r_{2}, r_{3}$ and $r_{4}$, with visual axes separated by an angular distance $d$. One of the sampling units sums signals from $r_{1}$ and $r_{2}$, weighted by factors $a$ and $(1-a)$ respectively. The other sampling unit sums signals from $r_{3}$ and $r_{4}$, weighted by the factors $(1-a)$ and $a$ respectively. The quantity $a(0<a<1)$ defines the relative weighting of signals from the two retinular cells that feed into each sampling unit. When $a=0$, only $r_{2}$ and $r_{3}$ are effective; when $a=1$, only $r_{1}$ and $r_{4}$ are effective. At crossover, signals from the two sampling units should be $180^{\circ}$ out of phase. Using this condition, it can be calculated that the crossover spatial frequency $v_{c}$ of this example obeys the relation

$\left[\frac{1}{2}-2 a \sin ^{2}\left(\pi v_{c} d\right)\right] \cos \left(\pi v_{c} d\right)=0$.

From this relation, it follows that for values of $a$ in the range $0<a<\frac{1}{4}$, the smallest crossover frequency is $\frac{1}{2 d}$. For values of $a$ in the range $\frac{1}{4}<a<1$, the smallest crossover frequency is given by $\frac{1}{\pi d} \sin ^{-1}\left(\frac{1}{2 \sqrt{a}}\right)$, which decreases continuously as $a$ is increased.

When $a=1$, the smallest crossover frequency is $\frac{1}{6 d}$.

Finally, in model $d$ (not illustrated), there is a large number of arrays. Each array only functions over a small range of luminances, and has the characteristics corresponding to that range. As luminance is varied, operation switches from one array to the next, but the changes between successive arrays are sufficiently small that it is experimentally difficult to detect the presence of more than one array at any given luminance.

In summing up this discussion of neural mechanisms, we can say that the available evidence argues against model $a$, but does not allow us to decide between models $b, c$ and $d$, or variations thereof.

\section{Functional Significance}

It is worthwhile to enquire into the functional significance of the characteristics of the movement detecting pathway. We do this by comparing these characteristics with those of an ideal movement detector designed for optimum performance.

Consider the nature of the movements that are typically encountered by the HI DSMD. The neuron is maximally sensitive to movement in the frontal field of view of the eye (Bishop et al., 1968; Eckert, 1980). Consequently, during free flight, it would monitor movements of the environment that occur within the frontal visual field. During straight-ahead flight, there would be virtually no motion in the frontal field, particularly when objects within this field are a fair distance away from the fly (Collett and King, 1975). When visually-guided course control is in operation, is is likely that excursions of the visual environment seen by the frontal field are restricted in amplitude, because the visual feedback loop will continuously work to stabilize the animal's orientation relative to its environment. Thus, if the environment suddenly begins to rotate relative to the animal's eyes, the extent to which its image slips across the retina would be limited by the velocity of the image and the reaction time of the control loop. Studies of visual fixation behaviour in flies clearly demonstrate that excursions of the fixated target are restricted in amplitude (rev. Reichardt and Poggio, 1976; see also Fig. 21 a).

Presuming that the role of the HI DSMD is to detect restricted, horizontal movements of the visual environment, how should it be designed for optimum performance? The answer to this question can be simplified by considering the design problem in two parts. We ask: (a) how should visual signals be filtered 


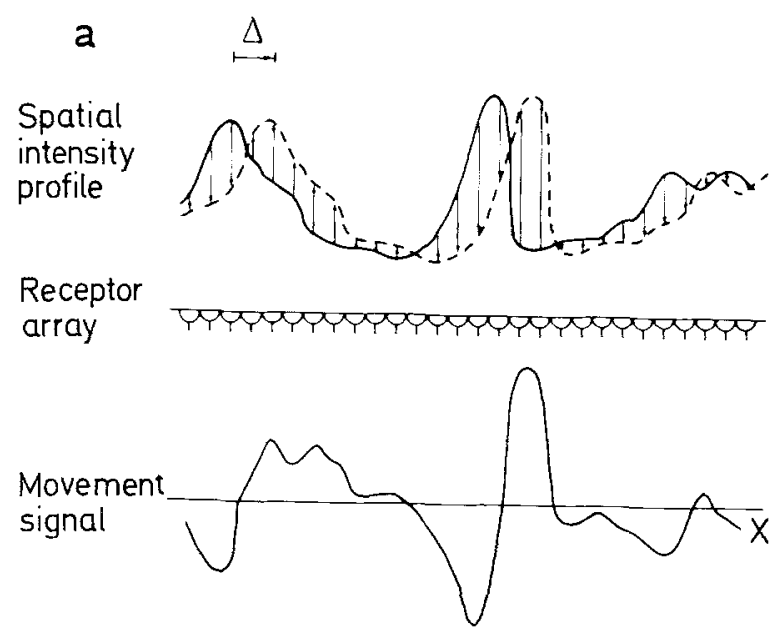

b

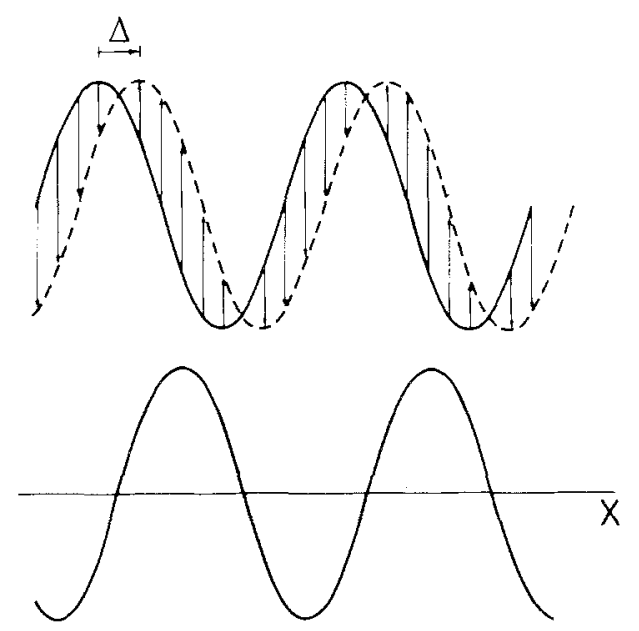

c
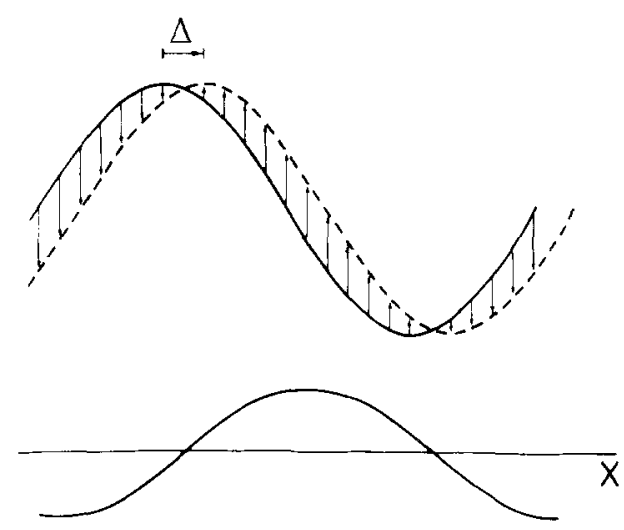

Fig. 20 a-c. Movement signals created across the receptor array when a visual pattern is instantaneously displaced by an angle of $4^{\circ}$. a Pattern with a spatially random intensity profile. $\mathbf{b}, \mathbf{c}$ Patterns with sinusoidal intensity profiles of spatial frequency $\frac{1}{64}$ and $\frac{1}{12 A}$ respectively. In each case, the upper solid curve shows the initial spatial intensity-profile of the pattern, the dashed curve shows the profile after displacement, and the lower curve shows the resulting movement signal, defined as the algebraic difference between the initial and displaced profiles (arrows) prior to movement detection, and (b) how should the filtered information be analyzed to produce a directional response?

Detection of movement will be most reliable (i.e. free of error) if the incoming visual signal is initially filtered so as to transmit only those features that provide reliable movement cues, and suppress those that do not. Which features of the visual environment - or, equivalently, which spatial frequencies - are the most reliable?

In order to answer this question, imagine that the array of photoreceptors in the eye views a random visual pattern with a spatial intensity profile as shown by the solid curve of Fig. 20a. Consider an instantaneous displacement of this pattern to a new position, $\Delta^{\circ}$ away, as shown by the dashed curve. The stimulus received by the photoreceptor array - and therefore by the movement-detecting system - will be the algebraic difference between the new intensity profile and the original one. The effective stimulus, or "movement signal", is indicated by the arrows in Fig. 20a, and redrawn separately below it. This is the signal that the visual system must use to detect motion and determine direction.

On examining the movement signal, it becomes apparent that low spatial frequencies of the visual environment make a relatively small contribution to it. If the size of the displacement is $\Delta^{\circ}$, then for all spatial frequencies lower than $\frac{1}{2 \Delta}$, the lower the frequency, the smaller the contribution. This property is illustrated in Fig. 20 b, c, which compare the movement signals produced by displacing sinusoids of spatial frequency $\frac{1}{6 A}$ and $\frac{1}{12 A}$, by $\Delta^{\circ}$.

By Fourier-analyzing the movement signal produced by displacing a spatially random pattern, one can calculate the extent to which various spatial frequencies of the retinal image contribute to the detection of movement. This is done in Appendix B, where we derive the "movement signal spectrum", which specifies the power in the movement signal at various spatial frequencies. The derivation assumes that displacements are random in size, with sizes distributed in gaussian fashion as they apparently are during visually-guided flight (Fig. 21 a).

The series of movement signal spectra in Fig. $21 \mathrm{~b}$ have been calculated for random gaussian displacements with characteristic sizes of $10^{\circ}$ (uppermost curve), $20^{\circ}, 40^{\circ}$ and $100^{\circ}$ (lowest curve). This series of spectra illustrates three important aspects of the movement signal spectrum:

(i) The contribution from low spatial frequencies is restricted because large displacements occur less frequently than small ones. 
a
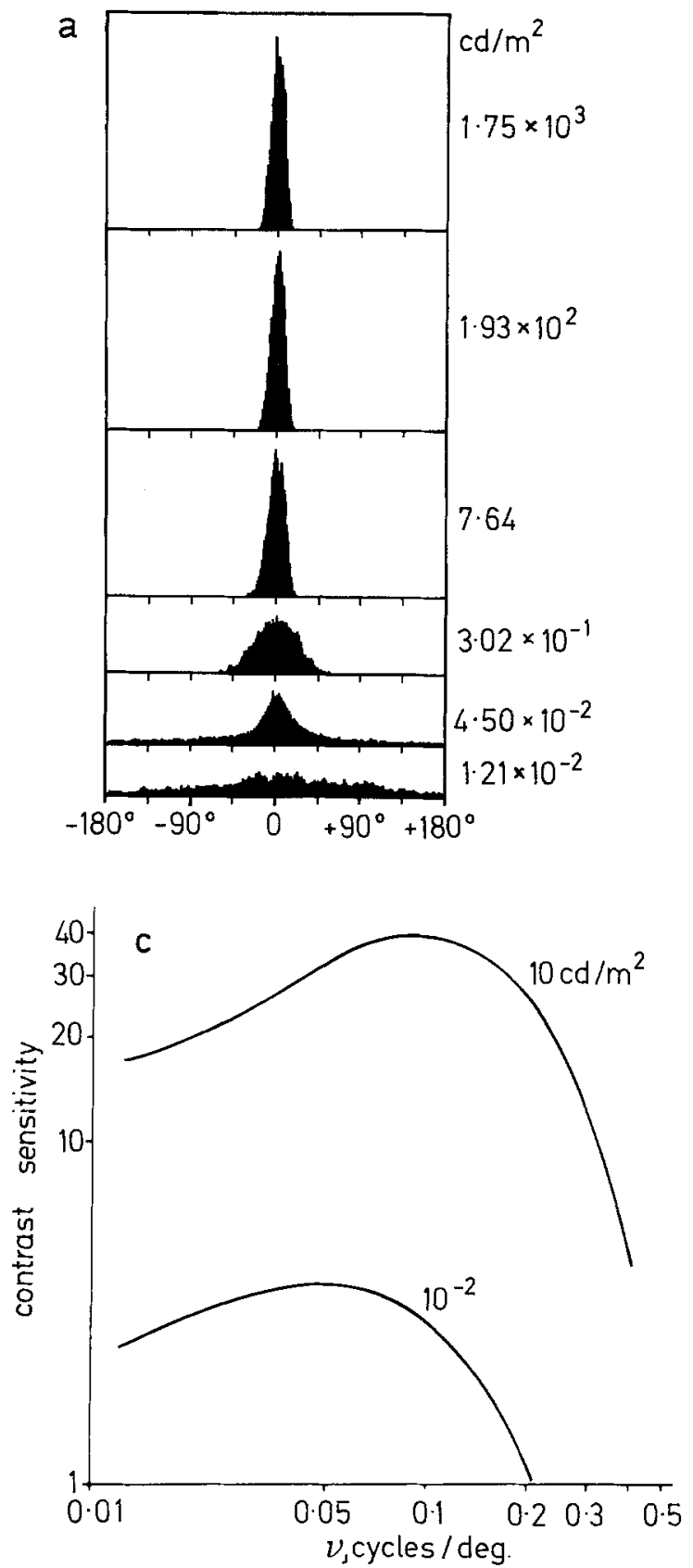
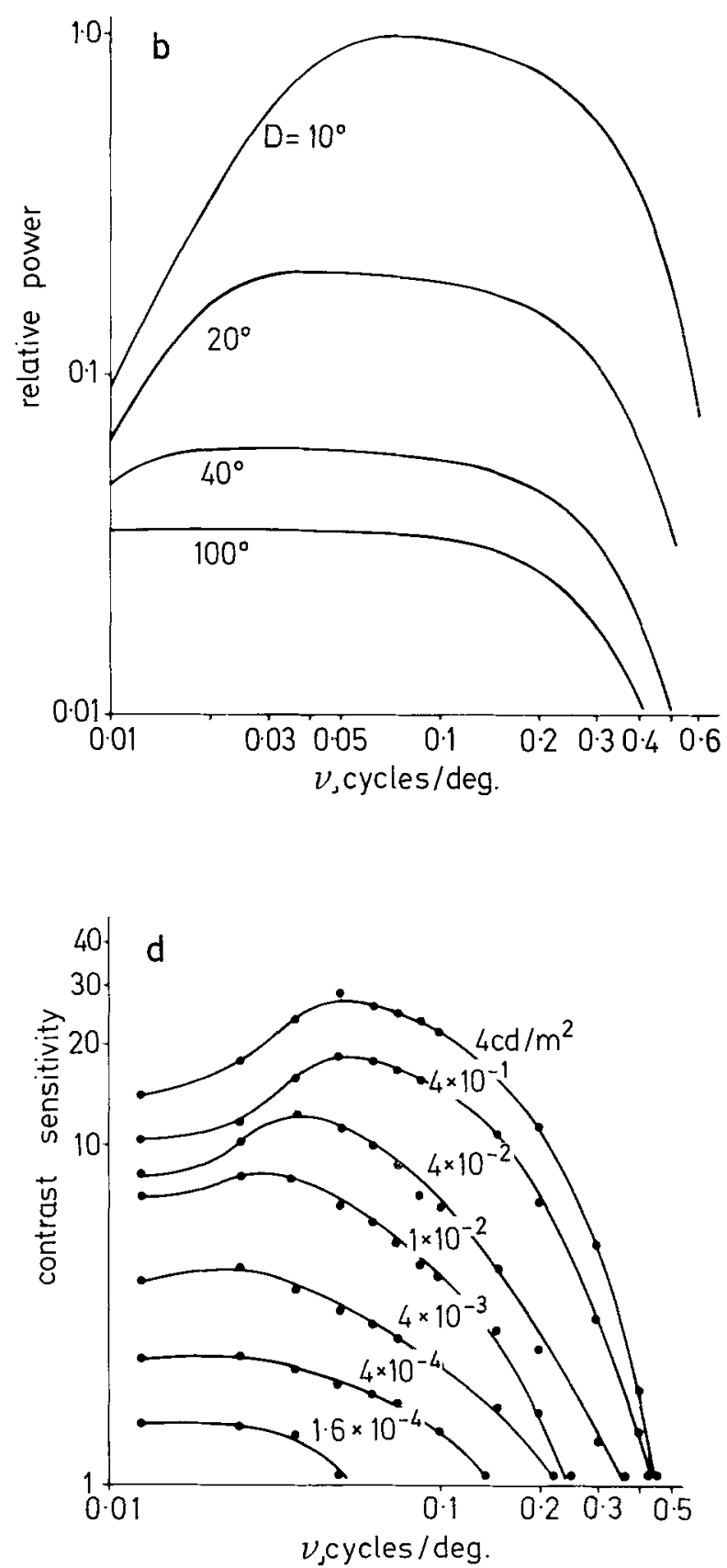

Fig. 21. a Orientation histograms, measured at various luminance levels, for a tethered fly (Musca) fixating a narrow vertical stripe in a closed-loop apparatus. This data has been redrawn from Fig. 5 of Reichardt and Poggio (1976), with the authors' permission, and experimental details can be found in Reichardt (1973). b Theoretical movement signal spectra, computed for a spatially random visual pattern undergoing displacements that are random in size, with sizes distributed in gaussian fashion, and with characteristic sizes as shown. For clarity, individual curves have been vertically shifted by arbitrary amounts. c Contrast-sensitivity functions of Hl DSMD, experimentally measured by using vertically-oriented reversing-contrast gratings, at luminances of $10 \mathrm{~cd} / \mathrm{m}^{2}$ (upper curve) and $0.01 \mathrm{~cd} / \mathrm{m}^{2}$ (lower curve). The curves shown are those that were used to approximate the data of Fig. 7 . d Contrast-sensitivity functions of HI DSMD, experimentally measured by using vertically-oriented gratings moving in the neuron's preferred direction at the indicated luminances. Data redrawn from Dvorak et al. (1980)

(ii) The contribution from high spatial frequencies is restricted by the lens, which acts as a spatial lowpass filter (Goodman, 1968; Snyder, 1979).

(iii) Intermediate spatial frequencies make the largest contribution to the movement signal. There- fore these frequencies are the most reliable, or noisefree, provided that the spatial power spectrum of the noise is uniform over all frequencies, i.e. flat (Snyder and Srinivasan, 1979). The movement-detecting system would function with the smallest probability of 
error if it works with these frequencies and not the other, less reliable ones.

Fig. $21 \mathrm{c}$,d shows experimentally measured contrast-sensitivity functions for comparison with the theoretically calculated movement signal spectra of Fig. 21 b. The behavioural data (Fig. 21 a) indicates that, at high levels of luminance, displacements of the visual scene are seldom larger than $10^{\circ}$. The movement signal spectrum corresponding to random gaussian displacements with a characteristic size of $10^{\circ}$ has its peak between 0.05 and 0.1 cycles/degree, and this coincides with the location of the peak of the experimentally measured contrast-sensitivity function (uppermost curves of Figs. $21 \mathrm{c}, \mathrm{d}$ ). As luminance is lowered, displacements become larger (Fig. 21 a). Presumably, at lower light levels, poorer signal-to-noise ratios in the visual system preclude detection (and correction) of smaller displacements. As the characteristic size of the displacements increases, the peak of the theoretical movement-signal spectrum progressively shifts toward lower spatial frequencies (Fig. 21 b). In other words, low spatial frequencies become increasingly important. Interestingly, the peak of the experimentalliy-measured contrast-sensitivity function tracks this shift (compare Fig. 21 b with d).

It would therefore seem that the peripheral stages of the movement-detecting pathway are designed to filter the visual signal in such a way as to transmit only those features (or spatial frequencies) that are rich in movement cues. At any given level of luminance, this spatial prefiltering "tunes" the movement detector to the frequencies that are most reliable, given the "expected" size of displacements. It was proposed earlier in the Discussion that, as the luminance is decreased, the changes in filtering properties arise from increased widths of the excitatory centres of the sampling units' receptive fields, and elimination of the inhibitory flanks.

We have discussed question (a), namely, how the retinal image should be filtered spatially to extract the richest movement cues. Let us now turn to question (b), and ask how the filtered signals, i.e. the outputs of the sampling units, should be analyzed to achieve maximum directional selectivity.

Fundamentally, the perception of direction of motion requires a comparison, or correlation, of signals that arise from two distinct regions of the visual scene (rev. Reichardt, 1969). In neuronal terms, this involves comparing signals from at least two sampling units as shown in Fig. 22. When a visual pattern moves toward the right, the signal from unit 1 will arrive at correlating site $B$ after being delayed by $\Delta$ t seconds, at about the same time as the signal from unit 2. Thus, correlator B will produce a response,
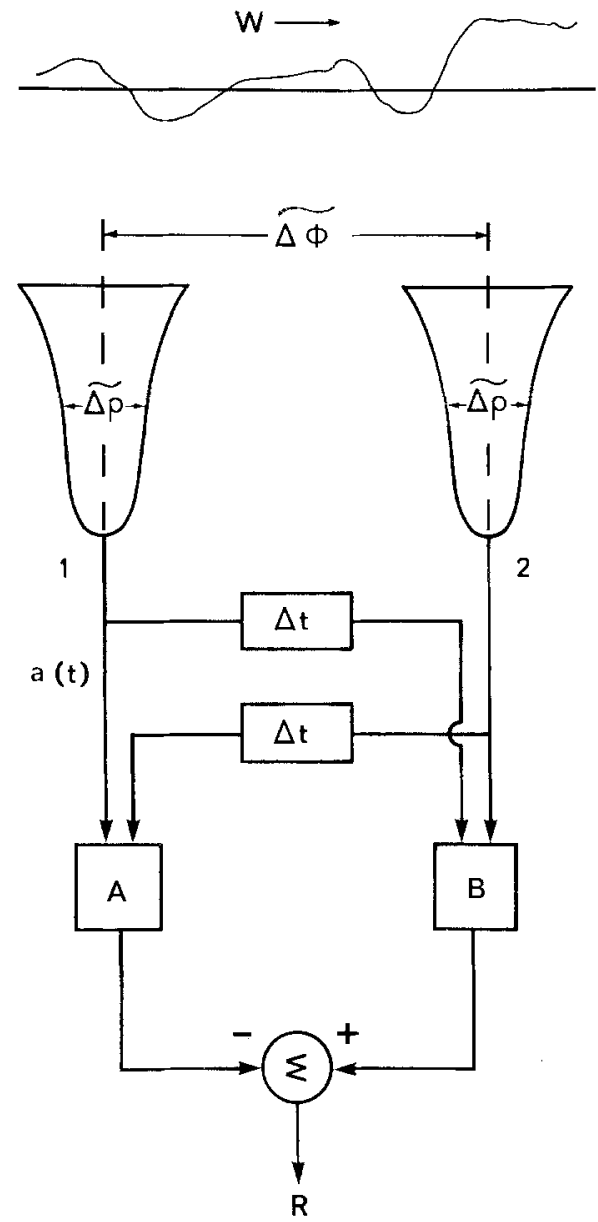

Fig. 22. Minimal schematic representation of a directionally-selective movement-detecting network. Details in text

causing the output $\mathrm{R}$ of the network to be positive. When the pattern moves toward the left, correlator A will produce a response, causing the output $\mathrm{R}$ to be negative. The network of Fig. 22 is a minimal schematic representation of any directionally-selective neuronal network, regardless of the physiological mechanisms that are actually employed (Barlow and Levick, 1965; Thorson, 1966; Poggio and Reichardt, 1976; Srinivasan and Bernard, 1976; Zaagman et al., 1978; Torre and Poggio, 1978).

We define directional selectivity as the algebraic difference between the responses evoked by moving a spatially random pattern toward the right and toward the left. What factors determine directional selectivity, and how can it be enhanced?

Let us assume that the half-width $\widetilde{\pi \rho}$ of the excitatory centre of a sampling unit is given (i.e. specified by the prefiltering requirements), and ask how large $\widetilde{\Delta \phi}$ should be to achieve maximum directional selectivity. In other words, how much overlap should there be between the receptive fields of a pair of correlating sampling-units? Assume for the mo- 
ment that the time delay $\Delta t$ and the speed $W$ of the pattern are fixed, and consider the effect of varying $\widetilde{\Delta \phi}$. If $\widetilde{\Delta \phi}$ is made arbitrarily large, directional selectivity will diminish to zero because the signals from the two sampling units will be uncorrelated and the network will not discriminate rightward from leftward motion. On the other hand, if $\widetilde{\Delta \phi}$ is made arbitrarily small, directional selectivity will again diminish to zero because the receptive fields of the sampling units will overlap to such an extent that the signals from the two units are identical and the two units become equivalent to one. Clearly, then, there is an optimum value of $\widetilde{\Delta \phi}$ which maximizes directional selectivity. In Appendix $\mathrm{C}$ we show that this optimum value is given by

$\widetilde{\Delta \phi}=\frac{\widetilde{\Delta \rho}}{1.67}$.

This relationship is independent of the speed of the pattern and the time delay $\Delta t$. Interestingly, the same relationship also minimizes the time delay $\Delta t$ that is required to achieve a given degree of directional selectivity (see Appendix C). The latter property may be important if the overall response latency of the DSMD is limited by the time delay $\Delta t$.

Maximum directional selectivity requires that there be substantial overlap between the receptive fields of neighbouring sampling stations, as specified by the above relation. We have inferred from measurements of the contrast sensitivity function (see earlier) that the value of $\widetilde{\Delta \rho}$ is $2.0^{\circ}$ at a luminance of $10 \mathrm{~cd} / \mathrm{m}^{2}$ and $3.1^{\circ}$ when the luminance is reduced by $3 \log$ units. Using the above relation, the theoretically optimum values of $\widetilde{\Delta \phi}$ corresponding to these values of $\widetilde{\Delta \rho}$ are $1.2^{\circ}$ and $1.8^{\circ}$ respectively. From the crossover experiments, the experimentally inferred values of $\widetilde{\Delta \phi}$ are $1.3^{\circ}$ and $1.7^{\circ}$ respectively. Thus, it appears that the optimum relationship between $\widetilde{\Delta} \rho$ and $\widetilde{\Delta \phi}$ is approximately achieved and maintained over a luminance range of at least $3 \log$ units, enabling the movement detector to maintain optimum directional selectivity over this range.

In conclusion, a comparison of theoretically-derived movement signal spectra with experimentallymeasured contrast sensitivity functions suggests that the movement detecting pathway prefilters the visual signal spatially in such a way as to extract reliable movement cues. The filtered information is then analyzed to detect movement in a way that achieves maximum directional selectivity.

\section{Role of Lateral Inhibition}

If the low-frequency attenuation of the contrast-sensitivity function is indeed due to lateral inhibition, as proposed earlier in the Discussion, then it appears that the role of lateral inhibition in this system is to suppress the low-spatial-frequency components of the visual scene - which are transmitted well by the lens, but provide relatively poor or unreliable movement cues - and to block the d.c. component, which bears no movement cues whatsoever. In other words, lateral inhibition filters out signals which are of little or no use in detecting movement.

DSMD neurons that are selective to horizontal motion apparently receive input from sampling units that have receptive fields with inhibitory flanks extending only along the horizontal direction (see earlier). This experimental finding corroborates the role proposed for lateral inhibition, because it is precisely what is expected on the basis of this role. Circularlysymmetrical surround inhibition - as in the centresurround organization of ganglion cells in the vertebrate retina - would be unnecessary in a system that is specialized to detect motion in a specific direction. It could even be detrimental, because the inhibition along the vertical direction would work against the excitation from the centre and reduce the modulation that would otherwise be produced by vertically-oriented features such as lines or edges, moving horizontally across the receptive field of each sampling unit. In this connection, it is interesting to note that movement detectors which have more than one preferred direction seem to be associated with sampling units that have "all-around" inhibition (Fig. 9).

\section{Movement Detection Versus Form Perception}

The criteria for designing a system that detects movement are not necessarily similar to those for designing a system that perceives form. For example, a movement detector that is involved in visually-guided course control is merely required to report the occurrence of movement, reliably and promptly. It does not have to be able to discriminate one shape from another. By the same token, such a movement detector does not have to possess high spatial acuity. There is no a priori need to preserve or reconstruct fine details of the visual scene at successive neural levels, if this detail does not provide reliable movement cues. It would not be surprising, therefore, if a design based on form perception is different from one based on movement detection. For example, Götz (1965) calculates values of 0.62 to 0.88 for $\frac{\widetilde{\Delta \rho}}{\widetilde{\Delta \phi}}$, based on optimum imaging; Snyder et al. (1977) use the concept of maximum information capacity to derive values ranging from 1.1 to 2.0 , depending upon luminance and contrast. 
In perceiving form, the human visual system strives to reconstruct fine details of the visual scene - if their contrast is high - by using neural mechanisms to "deblur" the retinal image (Georgeson and Sullivan, 1975; Snyder and Srinivasan, 1979). The fly's movement detector, on the other hand, shows little evidence of operating in this fashion. Contrastsensitivity functions of the movement detector, measured using high criterion responses (i.e. with highcontrast gratings) are not dramatically different in shape from those measured using low criterion responses (Dvorak et al., 1980). Apparently, this system is not specialized to encode the visual scene faithfully, but rather to extract reliable movement cues from the retinal image.

It is becoming increasingly likely that in many visual systems, including that of man, the functions of movement detection and form perception are performed by separate subsystems working in parallel, each presumably optimized to perform its own special task.

We thank Kevin Downing and his group for maintaining the equipment, Chris Snoek for help with the figures, Kerrie Ruth for photography and Pauline Wallace and Pam Coote for typing the manuscript. This work benefited substantially from discussions with $\mathrm{Si}$ mon Laughlin, Allan Snyder and Steve Shaw, and it was enthusiastically encouraged by Adrian Horridge.

\section{Appendix A}

Consider a sampling unit with a receptive field which has an excitatory centre characterized by a two-dimensional gaussian function with half-widths $\widetilde{A \rho}_{e x}$ and $\widetilde{A \rho}_{e y}$ along the horizontal $(x)$ and vertical $(y)$ directions respectively. This receptive field is described by

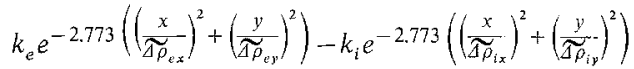

where the multiplying coefficients $k_{e}$ and $k_{i}$ specify the relative weighting of the excitatory and inhibitory zones, and where 2.773 $=4 \log _{e} 2$.

It can be shown that, for a receptive field specified by (A1), the contrast-sensitivity function along the horizontal direction (i.e. that measured with a vertically oriented grating) is given by

$C S F_{x}=k_{e} \widetilde{\Delta \rho_{e x}} \widetilde{\Delta \rho_{e y}} e^{-\left(\frac{\pi \widetilde{\Delta}_{e x} \nu_{x}}{1.67}\right)^{2}}-k_{i} \widetilde{\Delta \rho_{i x}} \widetilde{\Delta \rho_{i y}} e^{-\left(\frac{\pi \widetilde{\Delta}_{i x}{ }^{y} x}{1.67}\right)^{2}}$

and the contrast-sensitivity function along the vertical direction (i.e. that measured with a horizontally oriented grating) is given by

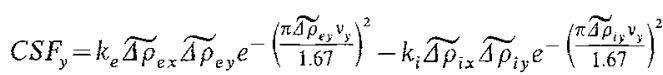

where $v_{x}$ and $v_{y}$ denote spatial frequencies along the $x$ and $y$ directions respectively, and a premultiplying factor of $\frac{2 \pi}{2.773}$ has been dropped, for simplicity. The calculation is similar to that of Enroth-Cugell and Robson (1966), except that their formulation assumes a circularly-symmetrical receptive field (i.e. $\widetilde{\Delta \rho}_{e x}=\widetilde{\Delta \rho}_{e y}$, and $\widetilde{T \rho}_{i x}=\widetilde{T \rho}_{i y}$ ) while ours does not.

Expressions (A2) and (A3) together contain 6 parameters: $k_{e}$, $k_{i}, \widetilde{A \rho}_{e x}, \widetilde{\Delta \rho}_{e y}, \widetilde{\Delta \rho}_{i x}$ and $\widetilde{T \rho}_{i y}$. A computer was programmed to adjust the values of these parameters to fit (A2) and (A3) to the contrast-sensitivity functions measured along the horizontal and vertical directions respectively (Fig. 7). The following parameter values yielded a least-squares fit. At high luminance $\left(10 \mathrm{~cd} / \mathrm{m}^{2}\right): k_{e}$ $=10.73, k_{i}=1.27,{\widetilde{\Delta \rho_{e x}}}=2.04^{\circ},{\widetilde{J \rho_{e y}}}_{e}=2.16^{\circ},{\widetilde{\Delta \rho_{i x}}}=10.52^{\circ}$ and $\widetilde{A \rho}_{i y}$ $=2.37^{\circ}$. At low luminance $\left(0.01 \mathrm{~cd} / \mathrm{m}^{2}\right): k_{e}=0.57, k_{i}=0.04, \widetilde{T}_{e}$ $=3.07^{\circ}, \widetilde{\Delta \rho}_{e y}=2.35^{\circ}, \widetilde{\Delta \rho}_{i x}=19.92^{\circ}$ and $\widetilde{\Delta \rho}_{i y}=2.40^{\circ}$.

The solid curves of Fig. 7 were plotted by substituting these parameter values into (A2) and (A3). The receptive-field profiles of Fig. 17 were obtained by substituting the parameter values into (A1) and setting $y=0$ for the horizontal profiles, and $x=0$ for the vertical profiles.

\section{Appendix B}

Here we derive expressions for the movement signal spectrum.

Displacements of Fixed Size

Consider a spatially random visual scene as in Fig. 20 a, described by the intensity profile $i(x)$, where $i$ denotes intensity and $x$ angular distance in degrees. The movement signal that is created when this profile is suddenly displaced by $\Delta^{\circ}$ is given by

$s(x)=i(x)-i(x-4)$

and its fourier transform is therefore (Bracewell, 1965):

$S(v)=I(v)\left[1-e^{-j 2 \pi v 4}\right]$

where $I(v)$ is the fourier transform of $i(x), v$ denotes spatial frequency in cycles per degree, and $j=\sqrt{-1}$.

The movement signal spectrum $M(v)$ is equal to $|S(v)|^{2}$. Using (B2), the movement signal spectrum is given by

$M(v)=4|I(v)|^{2} \sin ^{2}(\pi v \Delta)$.

In the above expression, $|I(v)|^{2}$ is the spatial power spectrum of the visual scene. Assuming that the scene is spatially random, $|I(v)|^{2}$ is equal to a constant, independent of $v$. Here we assume for simplicity (and without any loss of generality), that the constant is equal to $\frac{1}{4}$, so that

$M(v)=\sin ^{2}(\pi v \Delta)$.

Displacements of Random Size

Assume that displacements are random in size, with size specified by the probability density function $P(\Delta)$, where

$P(\Delta) \geqq 0, \quad-\infty<\Delta<\infty$

and

$\int_{-\infty}^{\infty} P(\Delta)=1$

The movement signal spectrum is then given by

$M(v)=\int_{\infty}^{\infty} P(\Delta) \sin ^{2}(\pi v \Delta) d \Delta$

where the function $\sin ^{2}(\pi v \Delta)$ describes the movement signal spectrum corresponding to a displacement of size $\Delta$.

For displacements that are random in size, with sizes distri- 
buted in gaussian fashion with characteristic size $D$, we have

$$
P(\Delta)=\frac{1}{\sqrt{\pi} D} e^{-\left(\frac{\Delta}{D}\right)^{2}} \quad(-\infty<\Delta<\infty)
$$

and (B5) can be written

$$
M(v)=\frac{1}{\sqrt{\pi} D} \int_{-\infty}^{\infty} e^{-\left(\frac{\Delta}{D}\right)^{2}} \sin ^{2}(\pi v \Delta) d \Delta
$$

which, upon integration, gives

$M(v)=1-e^{-(\pi v D)^{2}}$

where a premultiplying constant of $\frac{1}{2}$ has been dropped, for simplicity.

The spectra of (B4) and (B6) refer to the visual scene external to the eye. The movement signal spectrum at the level of the retina is obtained by multiplying these spectra by $|L(v)|^{2}$, where $L(v)$ is the modulation transfer function of the lens. We use Snyder's formula for the modulation transfer function of the lens of an ommatidium (Snyder, 1979, p. 229):

$L(v)=e^{-3.56\left[\frac{180}{\pi} \frac{\lambda v}{d}\right]^{2}}$

where $\lambda$ is the wavelength of light (microns) and $d$ the diameter of the aperture of the ommatidium (microns).

Assuming $\lambda=0.5 \mu$ and $d=30 \mu$, (B 7) can be approximately written

$L(v)=e^{-3.56 v^{2}}$.

Thus, the movement signal spectrum at the retina is given by

$M(v)=e^{-7.12 v^{2}}\left[\sin ^{2}(\pi v \Delta)\right]$

for a displacement of fixed size $\Delta$, and

$M(v)=e^{-7.12 v^{2}}\left[1-e^{-(\pi v D)^{2}}\right]$

for random-size displacements distributed in gaussian fashion with characteristic size $D$.

Expression (B9) was used to compute the series of spectra of Fig. 21 b.

\section{Appendix C}

Here we derive the relationship between $\widetilde{\Delta \phi}$ and $\widetilde{\Delta \rho}$ that maximizes the directional selectivity of the movement detector. We assume that (i) the schematic model of Fig. 22 is an accurate functional description of the movement-detecting process, (ii) each sampling unit has a gaussian receptive-field profile that is purely excitatory, with angular half-width $\widetilde{\Delta \rho}$ (it can be shown that the inclusion of inhibitory flanks, with strengths and dimensions as inferred from the data, affects the final result by less than $5 \%$ ), (iii) the speed of the visual pattern is small enough that the temporal filtering properties of the sampling units can be ignored.

With reference to Fig. 22, consider a spatially random pattern, moving toward the right at constant angular velocity $W$. The output of correlator $A$ will be $R_{a}\left(\frac{\widetilde{\Delta \phi}}{W}+\Delta t\right)$, where $R_{a}(\tau)$ denotes the autocorrelation function of the response $a(t)$ of sampling unit 1 (rev. Reichardt, 1969). Similarly, the output of correlator $B$ will be $R_{a}\left(\frac{\widetilde{\Delta \phi}}{W}-\Delta t\right)$. Therefore the response of the DSMD neuron to a visual pattern moving toward the right with velocity $W$ will be

$R=R_{a}\left(\frac{\widetilde{\Delta \phi}}{W}+\Delta t\right)-R_{a}\left(\frac{\widetilde{\Delta \phi}}{W}-\Delta t\right)$

which can be approximated to
$R \simeq 2\left[\frac{d R_{a}(\tau)}{d \tau}\right]=\frac{\pi \Phi}{W} \cdot \Delta t$

where the derivative of the autocorrelation function is evaluated at $\tau=\frac{\widetilde{\Phi \phi}}{W}$. Similarly, it can be shown that the response of the DSMD neuron to a visual pattern moving toward the left with constant angular velocity $W$ is identical to (C1), except negative in sign that is, opposite in direction. Directional selectivity of the DSMD neuron, defined as the algebraic difference between the responses evoked by motion to the right and motion to the left, is equal to $2 R$. (C1) demonstrates that, to a first-order approximation, directional selectivity is proportional to time delay $\Delta t$. Directional selectivity per unit time delay wili be maximum when the quantity in square brackets is a maximum. This condition requires that the derivative of the autocorrelation function of $a(t)$ be maximum at $\tau=\frac{\widetilde{\Delta \phi}}{W}$

It can be shown that the autocorrelation function $R_{a}(\tau)$, normalized to unity at $\tau=0$, is

$R_{a}(\tau)=e^{-\left[\frac{1.67 W \pm}{\sqrt{2} \widetilde{\mathbb{A}_{\rho}}}\right]^{2}}$

By differentiating $(C 2)$ twice, it can be shown that the maximum value of the derivative of $R_{a}(\tau)$ occurs at

$\tau=\frac{\widetilde{\Delta \rho}}{1.67 W}$

Setting this equal to $\frac{\widetilde{\Delta \phi}}{W}$ (as required by (C1)), we get

$\widetilde{\Delta \phi}=\frac{\widetilde{\Delta \rho}}{1.67}$.

This relationship achieves maximum directional selectivity per unit time delay. In other words, it ensures maximum directional selectivity for any given time delay, or, conversely, ensures minimum time delay for any given degree of directional selectivity.

\section{References}

Arnett, D.W. : Spatial and temporal integration properties of units in first optic ganglion of dipterans. J. Neurophysiol. 35, 429-444 (1972)

Barlow, H.B., Levick, W.R.: The mechanism of directionally selective units in the rabbit's retina. J. Physiol. 178, 477-504 (1965)

Beersma, D.G.M., Stavenga, D.G., Kuiper, J.W.: Organization of visual axes in the compound eye of the fly Musca domestica and behavioural consequences. J. Comp. Physiol. 102, 305-320 (1975)

Bishop, L.G.: A search for color encoding in the responses of a class of fly intemeurons. Z. Vergl. Physiol. 64, 355-371 (1969)

Bishop, L.G., Keehn, D.G.: Neural correlates of the optomotor response in the fly. Kybernetik 3, 288-295 (1967)

Bishop, L.G., Keehn, D.G., McCann, G.D.: Studies on motion detection by interneurons of the optic lobes and brain of the flies Calliphora phaenicia and Musca domestica. J. Neurophysiol. 31, 509-525 (1968)

Bracewell, R.: The Fourier transform and its applications. New York: McGraw-Hill (1965)

Braitenberg, V.: Periodic structures and structural gradients in the visual ganglia of the fly. In: Information processing in the visual systems of arthropods. Wehner, R. (ed.), pp. 3-15. Berlin, Heidelberg, New York: Springer 1972

Buchner, E.: Elementary movement detectors in an insect visual system. Biol. Cybern. 24, 85-101 (1976)

Buchner, E., Buchner, S., Hengstenberg, R.: Deoxy-glucose maps movement-specific nervous activity in visual ganglion of Drosophila. Science 205, 687-688 (1979)

Collett, T., King, A.J.: Vision during flight. In: The compound 
eye and vision of insects. Horridge, G.A. (ed.), pp. 437-466. Oxford: Clarendon 1975

Collett, T.S., Land, M.F.: Visual control of flight behaviour in the hoverfly, Syritta pipiens L. J. Comp. Physiol. 99, 1-66 (1975)

Dvorak, D.R., Bishop, L.G., Eckert, H.E.: On the identification of movement detectors in the fly optic lobe. J. Comp. Physiol. 100, 5-23 (1975)

Dvorak, D.R., Srinivasan, M.V., French, A.S. : The contrast sensitivity of fly movement-detecting neurons. Vision Res. 20, 397-407 (1980)

Eckert, H.: Functional properties of the H1-neurone in the third optic ganglion of the blowfly, Phaenicia. J. Comp. Physiol. 135, 29-39 (1980)

Eckert, H., Bishop, L.G. : Anatomical and physiological properties of the vertical cells in the third optic ganglion of Phaenicia sericata (Diptera, Calliphoridae). J. Comp. Physiol. 126, 57-86 (1978)

Enroth-Cugell, C., Robson, J.G.: The contrast sensitivity of retinal ganglion cells of the cat. J. Physiol. (London) 187, 517-552 (1966)

Gavel, L. von: Die "kritische Streifenbreite" als Mass der Sehschärfe bei Drosophila melanogaster. Z. Vergl. Physiol. 27, 80 135 (1939)

Geiger, G., Poggio, T.: The orientation of flies towards visual patterns: On the search for the underlying functional interactions. Biol. Cybern. 19, 39-54 (1975)

Georgeson, M.A., Sullivan, G.D.: Contrast constancy: deblurring in human vision by spatial frequency channels. J. Physiol. (London) 252, 627-656 (1975)

Goodman, J.W.: Introduction to Fourier optics. New York: McGraw-Hill 1968

Götz, K.G.: Behavioural analysis of the visual system of the fruitfly Drosophila. In: Proceedings of the symposium on information processing in sight sensory systems. Nye, P.W. (ed.). Pasadena: California Institute of Technology Press 1965

Hardie, R.C.: Peripheral visual function in the fly. Ph.D. Thesis, Australian National University, Canberra, Australia (1978)

Hausen, K.: Functional characterization and anatomical identification of motion sensitive neurons in the lobula plate of the blowfly Calliphora erythrocephala. Z. Naturforsch., Teil C 31, 628-633 (1976)

Hubel, D.H.: Tungsten microelectrode for recording from single units. Science 125, 549-550 (1957)

Kirschfeld, K.: The visual system of Musca: Studies on optics, structure and function. In: Information processing in the visual systems of arthropods. Wehner, R. (ed.), pp. 61-74. Berlin, Heidelberg, New York: Springer 1972

Kirschfeld, K.: The visual system of the fly: physiological optics and functional anatomy as related to behaviour. In: The neurosciences: Fourth study program. Schmitt, F.O., Worden, F.G. (eds.), pp. 297-310. Massachusetts: MIT Press 1978

Laughlin. S.B.: Neural principles in the peripheral visual systems of invertebrates. In: Handbook of sensory physiology, Vol. VII/6B. Autrum, H. (ed.), pp. 133-280. Berlin, Heidelberg, New York: Springer (1980)

Laughlin, S.B., Hardie, R.C.: Common strategies for light adaptation in the peripheral visual systems of fly and dragonfly. J. Comp. Physiol. 128, 319-340 (1978)

Lillywhite, P.G., Dvorak, D.R.: Responses to single photons in a fly optomotor neurone. Vision Res. (in press)

Marmarelis, P.Z., McCann, G.D.: Development and application of white-noise modeling techniques for studies of insect visual nervous system. Kybernetik 12, 74-89 (1973)

McCann, G.D.: The fundamental mechanism of motion detection in the insect visual system. Kybernetik 12, 64-73 (1973)

McCann, G.D.: Nonlinear identification theory models for successive stages of visual nervous systems of flies. J. Neurophysiol. 37, 869-895 (1974)
McCann, G.D., Arnett, D.W.: Spectral and polarization sensitivity of the dipteran visual system. J. Gen. Physiol. 59, 534-558 (1972)

Mimura, K.: Analysis of visual information in lamina neurones of the fly. J. Comp. Physiol. 88, 335-372 (1974)

Pick, B.: Visual pattern discrimination as an element of the fly's orientation behaviour. Biol. Cybern. 23, 171-180 (1976)

Pick, B., Buchner, E.: Visual movement detection under lightand dark-adaptation in the fly, Musca domestica. J. Comp. Physiol. 134, 45-54 (1979)

Pierantoni, R.: A look into the cock-pit of the fly. The architecture of the lobula plate. Cell Tissue Res. 171, 101-122 (1976)

Poggio, T., Reichardt, W.: Visual control of orientation behaviour in the fly. Part 2. Q. Rev. Biophys. 9, 377-438 (1976)

Reichardt, W.: Movement perception in insects. In: Processing of optical data by organisms and by machines. Reichardt, W. (ed.), pp. 465-493. New York: Academic Press 1969

Reichardt, W.: The insect eye as a model for analysis of uptake, transduction and processing of optical data in the nervous system. In: The neurosciences, Second study program. Schmitt, F.O. (ed.), pp. 494-510. New York: Rockefeller University Press 1970

Reichardt, W.: Musterinduzierte Flugorientierung. Naturwissenschaften 60, 122-138 (1973)

Reichardt, W., Poggio, T.: Visual control of orientation behaviour in the fly. Part 1. Q. Rev. Biophys. 9, 311-375 (1976)

Shaw, S.: Signal transmission by graded slow potentials in the arthropod peripheral visual system. In: The neurosciences: Fourth study program. Schmitt, F.O., Worden, F.G. (eds.) pp. 275-295. Massachusetts: MIT Press 1978

Snyder, A.W.: The physics of vision in compound eyes. In: Handbook of sensory physiology, Vol. VII/6A. Autrum, H. (ed.), pp. 225-313. Berlin, Heidelberg, New York: Springer 1979

Snyder, A.W., Srinivasan, M.V.: Human psychophysics: Functional interpretation for contrast sensitivity versus spatial frequency curve. Biol. Cybern. 32, 9-17 (1979)

Snyder, A.W., Stavenga, D.G., Laughlin, S.B.: Information capacity of compound eyes. J. Comp. Physiol. 116, 183-207 (1977)

Srinivasan, M.V., Bernard, G.D.: A proposed mechanism for multiplication of neural signals. Biol. Cybern. 21, 227-236 (1976)

Strausfeld, N.J. : Mosaic organizations, layers, and visual pathways in the insect brain. In: Neural principles in vision. Zettler, F., Weiler, R. (eds.), pp. 245-279. Berlin, Heidelberg, New York: Springer 1976

Thorson, J.: Small-signal analysis of a visual reflex in the locust. Part 2. Kybernetik 3, 53-66 (1966)

Torre, V., Poggio, T.: A synaptic mechanism possibly underlying directional selectivity to motion. Proc. R. Soc. (London) Ser. B 202, 409-416 (1978)

Wyszecki, G., Stiles, W.S. : Color science. New York: John Wiley 1967

Zaagman, W.H., Mastebroek, H.A.K., Buyse, T., Kuiper, J.W. Receptive field characteristics of a directionally selective movement detector in the visual system of the blowfly. J. Comp. Physiol. 116, 39-50 (1977)

Zaagman, W.H., Mastebroek, H.A.K., Kuiper, J.W.: On the correlation model: Performance of a movement detecting neural element in the fly visual system. Biol. Cybern. 31, 163-168 (1978)

Zettler, F., Järvilehto, M. : Intraaxonal visual responses from visual cells and second-order neurons of an insect retina. In : Information processing in the visual systems of arthropods. Wehner, R. (ed.), pp. 217-222. Berlin, Heidelberg, New York: Springer 1972

Zettler, F., Weiler, R.: Neuronal processing in the first optic neuropile of the compound eye of the fly. In: Neural principles in vision. Zettler, F., Weiler, R. (eds.), pp. 227-236. Berlin, Heidelberg, New York: Springer 1976 